\title{
Polygenicity and epistasis underlie fitness-proximal traits in the Caenorhabditis elegans multiparental experimental evolution (CeMEE) panel
}

\author{
Luke M. Noble*,1, Ivo Chelo ${ }^{\dagger}$, Thiago Guzella $\$$, Bruno Afonso ${ }^{\S}{ }^{\dagger}$, David D. Riccardi*, Patrick Ammerman*, Adel Dayarian**, Sara \\ Carvalho $^{\dagger}$, Anna Crist ${ }^{\S}$, Ania Pino-Querido ${ }^{\dagger}$, Boris Shraiman ${ }^{* *,}$, Matthew V. Rockman ${ }^{* 1}$ and Henrique Teotónio ${ }^{\S}, 1$ \\ ${ }^{*}$ Center for Genomics and Systems Biology, Department of Biology, New York University, New York, NY, 10003, USA, 'Instituto Gulbenkian de Ciência, Oeiras, \\ Portugal, §Institut de Biologie, École Normale Supérieure, CNRS UMR 8197, INSERM U1024, F-75005 Paris, France, ${ }^{* *}$ Kavli Institute for Theoretical Physics \\ and, $\ddagger$ Department of Physics, University of California, Santa Barbara, CA, 93106, USA
}

\begin{abstract}
Understanding the genetic basis of complex traits remains a major challenge in biology. Polygenicity, phenotypic plasticity and epistasis contribute to phenotypic variance in ways that are rarely clear. This uncertainty is problematic for estimating heritability, for predicting individual phenotypes from genomic data, and for parameterizing models of phenotypic evolution. Here we report a recombinant inbred line (RIL) quantitative trait locus (QTL) mapping panel for the hermaphroditic nematode Caenorhabditis elegans, the $C$. elegans multiparental experimental evolution (CeMEE) panel. The CeMEE panel, comprising 507 RILs, was created by hybridization of 16 wild isolates, experimental evolution at moderate population sizes and predominant outcrossing for 140-190 generations, and inbreeding by selfing for $13-16$ generations. The panel contains $22 \%$ of single nucleotide polymorphisms known to segregate in natural populations, and complements existing mapping resources for C. elegans by providing high nucleotide diversity across $>95 \%$ of the genome. We apply it to study the genetic basis of two fitness components, fertility and hermaphrodite body size at time of reproduction, with high broad sense heritability in the CeMEE. While simulations show we should detect common alleles with additive effects as small as $5 \%$, at gene-level resolution, the genetic architectures of these traits does not feature such alleles. We instead find that a significant fraction of trait variance, particularly for fertility, can be explained by sign epistasis with weak main effects. In congruence, phenotype prediction, while generally poor $\left(r^{2}<10 \%\right)$, requires modeling epistasis for optimal accuracy, with most variance attributed to the highly recombinant, rapidly evolving chromosome arms.
\end{abstract}

KEYWORDS genetic architecture; polygenicity; epistasis; experimental evolution; body size; fertility; selfing; GWAS; heritability; quantitative trait; complex trait; QTL; MPP

\section{Introduction}

Most measurable features of organisms vary among individuals. Outlining the genetic dimension of this variation, and how this varies across populations and traits, has important implications for the application of genomic data to predict disease risk and agricultural production, for estimation of heritability, and for understanding evolution (Lynch and Walsh 1998; Barton and Keightley 2002). Complex traits are defined by being multifactorial. They tend to be influenced by many genes and to be plastic in the presence of environmental variation, and the manner in which phenotypic variation emerges from the combined effects of causal alleles is rarely clear. Although phenotype prediction and some aspects of evolution can often be well approximated by considering additive effects alone, non-additive interactions between alleles at different loci (with marginal additive effects) may explain a large fraction of trait variation yet remain undetected due to low statistical power (Phillips 2008). Adding further complication, one cannot usually assume that genetic and environmental effects are homogeneous or independent of one another (Barton and Turelli 1991; Félix and Barkoulas 2015), nor that the genetic markers used for mapping quantitative trait loci (QTL) are faithfully and uniformly associated with causal alleles (Yang et al. 2010; Speed et al. 2012). 
bioRxiv preprint doi: https://doi.org/10.1101/120865; this version posted March 27, 2017. The copyright holder for this preprint (which was not certified by peer review) is the author/funder, who has granted bioRxiv a license to display the preprint in perpetuity. It is made available under aCC-BY 4.0 International license.

INTRODUCTION

Human height, for example, is the canonical quantitative 103 trait, an easily measured, stable attribute with high heritability 104 (around 80\%) when measured in families Fisher (1930); Galton 105 (1886); Visscher et al. (2010). Hundreds of common QTL (minor 106 allele frequency, $\mathrm{MAF}>5 \%$ ) of small effect have been detected 107 by genome-wide association studies (GWAS) over the last two 108 decades, explaining in sum only a small fraction (around 20\%) 109 of heritability (Wood et al. 2014). A recent study with more 110 than $7 \times 10^{5}$ people showed that close to one hundred uncom- 111 mon QTLs $(0.1 \%<\mathrm{MAF}<5 \%)$ of more moderate effects explain a 112 mere extra 5\% of heritability (Marouli et al. 2017). It has taken ${ }_{113}$ methods of genomic selection in animal breeding, and dense 114 genetic marker information (Meuwissen et al. 2001; Meuwissen 115 and Goddard 2010), to show that common QTL of very small ${ }_{116}$ effect can potentially explain a large fraction of the variability in 117 human height and common diseases (Yang et al. 2010; Speed et al. 118 2016). Thus, in perhaps many cases, the so-called problem of 119 the "missing heritability" may be synonymous with high poly- 120 genicity (Hill et al. 2008; Manolio et al. 2009). The contribution of 121 statistical epistasis to variation in human height is likely to be ${ }_{122}$ modest (Visscher et al. 2010), although the generality of this for ${ }_{123}$ size-related traits in other organisms is not known. Molecular ${ }_{124}$ genetics and biochemistry suggest functional non-additivity is 125 ubiquitous within individuals, and significant effects on trait ${ }_{126}$ variation have been shown in many cases (e.g., MUKAI (1967); 127 Whitlock and Bourguet (2000); Bonhoeffer et al. (2004); Carlborg ${ }_{128}$ et al. (2006); de Visser et al. (2009); Zwarts et al. (2011); Shao et al. 129 (2008); Gaertner et al. (2012); Barkoulas et al. (2013); Weinreich 130 et al. (2013); Huang et al. (2014); Vanhaeren et al. (2014); Bloom 131 et al. (2015); Monnahan and Kelly (2015b,a); Paaby et al. (2015); 132 Tyler et al. (2016); Schoustra et al. (2016); Forsberg et al. (2017); Chirgwin et al. (2016), but the importance of epistasis in shaping fitness landscapes and in generating the additive genetic variance on which selection can act is still debated (Cheverud and Routman 1995; Wolf et al. 2000; Phillips 2008; Hansen 2013; Mackay et al. 2014)).

Alongside GWAS, inbred line crosses in model systems con- ${ }_{139}$ tinue to be instrumental for our understanding of the genetics of 140 complex traits, given the opportunity for control of confounding 141 environmental covariates and accurate measurement of breeding 142 values. Crosses among multiple parental strains in particular ${ }_{143}$ - such as those now available for mice (Churchill et al. 2004), ${ }_{144}$ Drosophila (Macdonald and Long 2007), maize (McMullen et al. 145 2009; Buckler et al. 2009), wheat (Huang et al. 2012; Mackay et al. 146 2014; Thepot et al. 2015), rice (Bandillo et al. 2013), tomato (Pas- 147 cual et al. 2015) and Arabidopsis (Kover et al. 2009), among others 148 - have been developed to better sample natural genetic variation. 149 Greater variation also allows the effects of multiallelic loci to 150 be studied and, subject to effective recombination, improved 151 QTL resolution. If large populations and random mating are ${ }_{152}$ imposed for long periods, gains in resolution can be dramatic ${ }_{153}$ (Valdar et al. 2006; Rockman and Kruglyak 2008), although this 154 comes at the expense of increased opportunity for selection to 155 purge diversity (e.g., Baldwin-Brown et al. (2014); Rockman and 156 Kruglyak (2009)).

Better known as a model for functional biology (Corsi et al. 2015), the nematode Caenorhabditis elegans has also contributed to our understanding of complex traits and their evolution. $C$. elegans shows extensive variation in complex traits (Gems and Riddle 2000; Knight et al. 2001; Barrière and Félix 2005; Gutteling 1 et al. 2007; Gray and Cutter 2014; Diaz and Viney 2014; Teotónio 163 et al. 2017) and sex-determination and breeding mode (selfing and outcrossing) can be genetically manipulated at will. QTL for traits such as embryonic lethality (Rockman and Kruglyak 2009), pesticide resistance (Ghosh et al. 2012) and telomere length (Cook et al. 2016) have been found by association studies in an ever expanding panel of inbred wild isolates, the C. elegans natural diversity resource (CeNDR; https:/ / elegansvariation.org/, Cook et al. (2017)). QTL for a range of complex traits have also been found using collections of recombinant inbred lines (RILs) (Rockman and Kruglyak 2009) and introgression lines (ILs) (Doroszuk et al. 2009) derived from crossing the laboratory domesticated N2 strain (Sterken et al. 2015) and the divergent Hawaiian wild isolate CB4856 (e.g., Andersen et al. $(2014,2015))$, or by two-parent crossing of non-domesticated strains (e.g., Duveau and Félix (2012); Noble et al. (2015)). GWAS and two-parent crosses have given insights into how natural selection has shaped phenotypic variation in C. elegans and related nematodes. For example, an N2/CB4856 RIL panel has been used to argue that selection on linked sites largely explains the distribution of QTL effects for mRNA abundance (Rockman et al. 2010). Lastly, C. elegans is also one of the main models for experimental evolution (Gray and Cutter 2014; Teotónio et al. 2017). Mutation accumulation line panels in particular have long been used to estimate mutational heritability (Estes and Lynch 2003; Estes 2005; Baer et al. 2005; Baer 2008; Phillips et al. 2009; Halligan and Keightley 2009) and to argue that standing levels of genetic variation in natural populations for complex traits can be explained by a mutationselection balance (Etienne et al. 2015; Farhadifar et al. 2016). As yet, the QTL mapping resolution of existing C. elegans RIL panels has been coarse, and there is no panel derived from crosses of multiple wild parental strains.

A prominent characteristic of C. elegans is its mixed androdioecious reproductive system, with hermaphrodites capable of either selfing, from a cache of sperm produced late in larval development (Hirsh et al. 1976), or outcrossing with males (Maupas 1900). Sex determination is chromosomal, with hermaphrodites $X X$, and $X O$ males maintained through crosses and rare $X-$ chromosome non-disjunction during hermaphrodite gametogenesis (Nigon 1949). Because males are typically absent from selfed broods but are half the progeny of a cross, twice the male frequency in a population is the expected outcrossing rate (Stewart and Phillips 2002; Cutter 2004). Natural populations have low genetic diversity and very high linkage disequilibrium (LD), with generally weak global population structure and high local diversity among typically homozygous individuals at the patch scale (Barrière and Félix 2005, 2007; Cutter et al. 2009). Average single nucleotide polymorphism (SNP) diversity is on the order of $0.3 \%$ (Cutter 2006) though highly variable across the genome, reaching $16 \%$ or more in some hypervariable regions (Thompson et al. 2015). Low diversity and high LD is due to the predominance of inbreeding by selfing, which reduces the effective recombination rate and elevates susceptibility to linked selection (Rockman et al. 2010; Andersen et al. 2012). Crosses between wild isolates have revealed outbreeding depression (Dolgin et al. 2007; Chelo et al. 2014), which may be in part due to the disruption of epistatic allelic interactions. Evidence supporting this prediction in C. elegans is, to date, scarce: one study has shown that recombination between several QTL "complexes" leads to dysregulation of thermal preferences (Gaertner et al. 2012).

Although selfing is the most common reproductive mode in natural C. elegans populations, males, though rare, are variably proficient in mating with hermaphrodites (Teotónio et al. 2006; 
bioRxiv preprint doi: https://doi.org/10.1101/120865; this version posted March 27, 2017. The copyright holder for this preprint (which was not certified by peer review) is the author/funder, who has granted bioRxiv a license to display the preprint in perpetuity. It is made available under

Murray et al. 2011). Perhaps as a consequence of low but sig- 226 nificant outcrossing (and also a metapopulation demographic 227 structure) several loci have been found to be under some form of ${ }_{228}$ balancing selection (e.g., Ghosh et al. (2012); Greene et al. (2016)). 229 Moreover, evolution experiments involving crosses among mul- 230 tiple strains have shown that high outcrossing rates can persist ${ }_{231}$ as long as there is heritable variation for male traits (Anderson ${ }_{232}$ et al. 2010; Teotónio et al. 2012; Masri et al. 2013). In our evolu- 233 tion experiments in particular (Teotónio et al. 2012), moderate 234 population sizes and high outcrossing rates facilitated the loss of 235 genetic diversity by (partial) selective sweeps, with excess het- 236 erozygosity maintained by epistatic selection on overdominant ${ }_{237}^{23}$ loci (e.g., Chelo and Teotónio (2013); Chelo et al. (2014)).

This foundation suggests study of C. elegans may be fruit- 239 ful for our understanding of the contribution of within- and 240 between-locus interactions to complex traits and their evolu- ${ }_{241}$ tion. Here we present a panel of 507 genome sequenced RILs 242 obtained by intercrossing 16 wild isolates, culturing at high out- 243 crossing rates in populations of $\approx 10^{4}$ for 140-190 generations of ${ }_{244}$ experimental evolution, followed by inbreeding by selfing for ${ }_{245}$ 13-16 generations. The $\underline{C}$. elegans Multiparental Experimental 246 Evolution (CeMEE) RIL panel complements existing C. elegans ${ }_{247}$ mapping resources by providing fine mapping resolution and 248 high nucleotide diversity. Using simulations, we show that the ${ }_{249}$ CeMEE panel can give gene-level resolution for common QTL ${ }_{250}$ with effects as low as $5 \%$. In subsets of the CeMEE, we investigate the genetic basis of two fitness components, fertility and hermaphrodite body size at the time of reproduction, by variance decomposition under additive and additive-by-additive ${ }^{252}$ epistatic models, and by genome-wide 1- and 2-dimensional 253 association testing. We find that the genetic basis of both traits, 254 particularly fertility, is highly polygenic, with a significant role 255 for epistasis.

\section{Materials and Methods}

\section{CeMEE derivation}

The panel was derived in 3 stages (Figure 1). First, 16 wild ${ }^{261}$ isolates (AB1, CB4507, CB4858, CB4855, CB4852, CB4586, MY1, 262 MY16, JU319, JU345, JU400, N2 (ancestral), PB306, PX174, PX179, 263 RC301; obtained from the Caenorhaditis Genetics Center) were 264 inbred by selfing for 10 generations to ensure homozygosity, 265 then intercrossed to funnel variation into a single multiparental 266 hybrid population, as described in Teotónio et al. (2012). Each of 267 the four funnel phases comprised multiple pairwise, reciprocal 268 crosses at moderate population sizes (see Figure S1 of Teotónio 269 et al. (2012) for full details of replication and population sizes). 270

Second, the multiparental hybrid population was evolved 271 for 140 discrete generations at population sizes of $N \approx 10^{4}$ (out- 272 crossing rate $\left.\approx 0.5, N_{e} \approx 10^{3}\right)$, to obtain the $\mathrm{A} 140$ population, as 273 reported in (Teotónio et al. 2012; Chelo and Teotónio 2013; Chelo 274 et al. 2013). Sex-determination mutations were then mass intro- 275 gressed into the A140, while maintaining genetic diversity, to 276 generate monoecious (obligately selfing hermaphrodites) and tri- 277 oecious (partial selfing with males, females and hermaphrodites) 278 populations, as detailed in Theologidis et al. (2014). Further 279 replicated experimental evolution was carried out for 50 gener- 280 ations under two environmental regimes: (1) a Control regime 281 (conditions as before), with the wild-type Androdioecious re- 282 productive system (CA50 collectively, full designations can be 283 found in Table S1); and (2) a Gradual exposure to an increasing 284 gradient of $\mathrm{NaCl}$, from $25 \mathrm{mM}$ (standard NGM-lite medium, US 285 Biological) to $305 \mathrm{mM}$ until generation 35 and thereafter, vary- 286 ing reproductive system (GX50, where $X$ is Androdioecious, Monoecious or Trioecious). Although trioecious populations started evolution with only $0.1 \%$ of hermaphrodites, by generation 50 they were abundant (50\%; see Figure S7 in Theologidis et al. (2014)). Androdioecious populations maintained outcrossing rates of $>0.4$ until generation 35 , soon after losing males to finish with an outcrossing rate of about 0.2 by generation 50 (Figure S5 in Theologidis et al. (2014)). The effects of reproductive system on the genetics and evolution of complex traits will be the subject of future work.

Finally, hermaphrodites were inbred by selfing to obtain recombinant inbred lines (RILs). Population samples $\left(>10^{3}\right.$ individuals) were thawed from -80C and maintained under standard laboratory conditions for two generations. At the third generation, single hermaphrodites were picked at the late third to early fourth (L3/L4) larval stage and placed in wells of 12-well culture plates, containing M9 medium $(25 \mathrm{mM} \mathrm{NaCl})$ seeded with E. coli. Lines were propagated at $20 \mathrm{C}$ and $80 \% \mathrm{RH}$ by transferring a single L3/L4 individual for 16 (A140 population) or 13 generations (4-7 days between transfers). At each passage, parental plates were kept at $4 \mathrm{C}$ to prevent growth until offspring production was verified, and in the case of failure a second transfer was attempted before declaring line extinction. Inbreeding was done in several blocks from 2012 to 2016, in two different labs. A total of 709 RILs were obtained and archived at -80C (File S2).

\section{Sequencing and genotyping}

DNA of the 16 founders, 666 RILs and the A140 population was prepared using the Qiagen Blood and Tissue kit soon after derivation or after thawing from frozen stocks and expansion to at least $10^{4} \mathrm{~L} 1$ individuals. Founders were sequenced to $>=30 \mathrm{X}$ depth with 50 or 100bp paired-end reads (Illumina HiSeq 2000, New York University Center for Genomics and Systems Biology GenCore facility). Reads were mapped (BWA 0.7.8; Li and Durbin (2010)) to the WS220 C. elegans N2 reference genome and variants (SNPs and small indels) were called jointly (GATK 3.3-0 HaplotypeCaller; McKenna et al. (2010)), followed by base quality score recalibration (BQSR) using a subset of high scoring sites $(29 \%$ of initial variants passing strict variant filtration: " $\mathrm{MQ}<58.0$ । । $\mathrm{DP}<20$ | | FS $>40.0$ | | SOR $>3.0$ | | ReadPosRankSum $<-5.0$ | | QD $<20.0$ | | DP $>$ mean $\times 2$ "). Diallellic single nucleotide variants on the six nuclear chromosomes were intersected with calls from a joint three-sample call (GATK UnifiedGenotyper) on pooled founders, a subset of pooled RILs (SUP TABLE XX, SAME AS CEMEE LIST ANOTHER COLUMN), and 72X sequencing of the A140 population (approximately 1400x total), then filtered based on variant call metrics $(\mathrm{MQ}<50.0|| \mathrm{DP}<$ 10 | | FS > 50.0 | | SOR > 5.0 । | ReadPosRankSum <-5.0 | | $\mathrm{QD}<6.0|| \mathrm{DP}>$ mean $\times 3$ ) and on the number of heterozygous or missing founder calls (3,014 sites $>8$ removed; these calls likely represent copy number differences between founders and the N2 reference), and requiring $\geqslant 1$ homozygote $(28,872$ sites removed), giving an initial set of 404,536 SNP markers.

RILs were sequenced with 100bp paired-end reads (Nextera libraries, HiSeq 2000, NYU) or 150bp paired-end reads (Hiseq $X$ Ten, BGI Tech Solutions Company, Hong Kong), to mean depth 7.2X (minimum 0.2X). Genotypes were imputed by Hidden Markov Model (HMM) considering the 16 founder states and mean base qualities of reads. Downsampled predictions for a subset of RILs sequenced to high (20-30X) depth gave imputation accuracy of approximately $99 \%$ at $0.2 \mathrm{X}$ and $99.9 \%$ at $0.5 \mathrm{X}$ ( $93 \%$ of lines). 


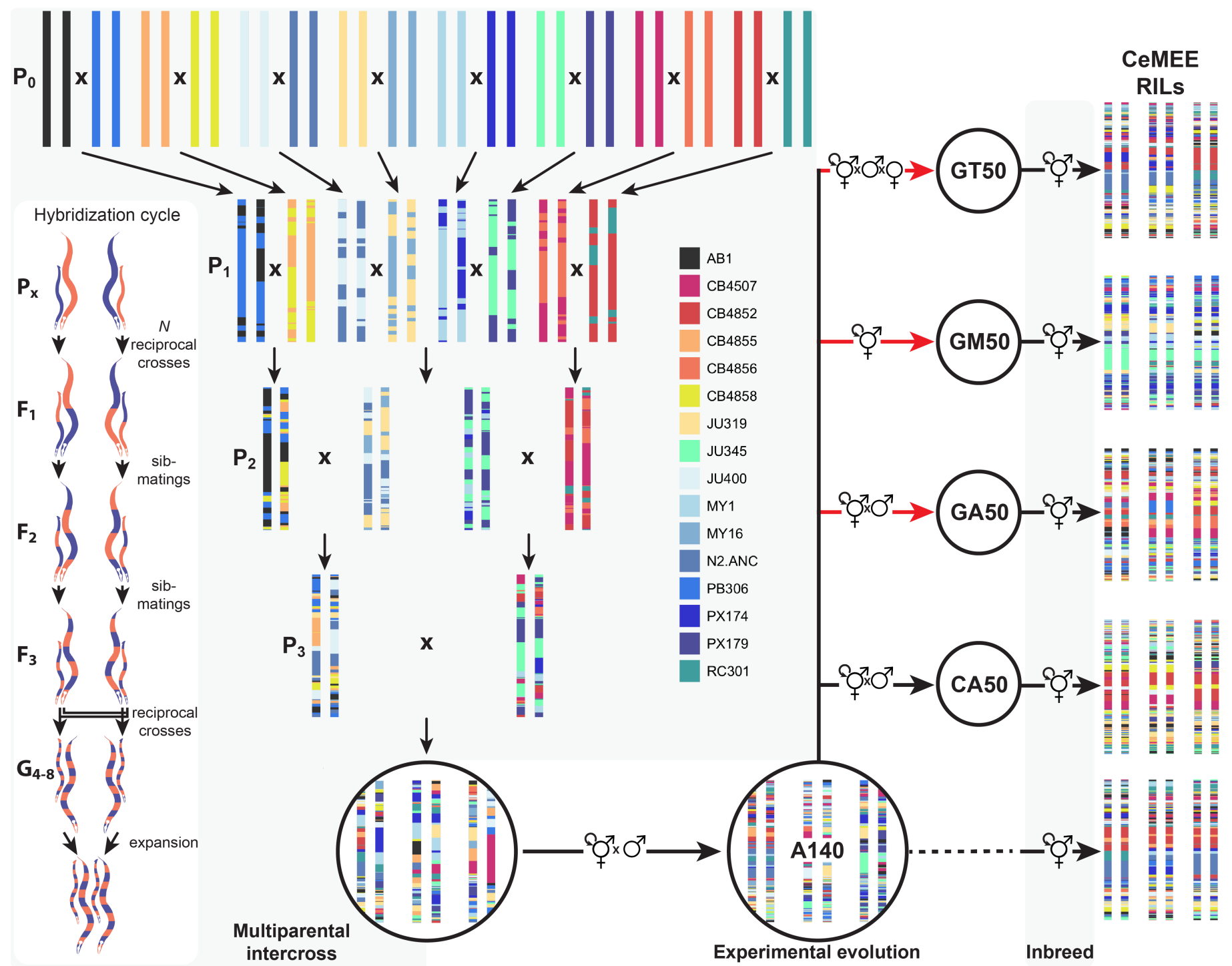

Figure 1 CeMEE derivation. The multiparental intercross funnel phase comprised four stages of pairwise crosses and progeny mixing, carried out in parallel at controlled population sizes. One hybridization cycle for a single founder cross is inset at left: in each cycle, multiple reciprocal crosses were initiated, increasing in replicate number and census size each filial generation. $F_{1}$ and $F_{2}$ progeny were first sib-mated, then reciprocal lines were merged by intercrossing the $F_{3}$ and expanding the pooled $G_{4}$ (for three to four generations) before commencing the next reduction cycle. The resulting multiparental hybrid population was archived by freezing, and samples were thawed and then maintained for 140 non-overlapping generations of mixed selfing and outcrossing under standard laboratory conditions to generate the A140 population. Hermaphrodites were then sampled from the A140 and selfed to generated the A140 RILs. Additionally, the outbred A140 population was evolved for a further 50 generations under the same conditions (control adapted lines; CA) or under adaptation to a salt gradient with varying sex ratios (GT, GM and GA lines; Theologidis et al. (2014)). See Materials and Methods for description of sub-panels, and Teotónio et al. (2012) for details of replicate numbers and population sizes for each funnel generation. 
bioRxiv preprint doi: https://doi.org/10.1101/120865; this version posted March 27, 2017. The copyright holder for this preprint (which was not certified by peer review) is the author/funder, who has granted bioRxiv a license to display the preprint in perpetuity. It is made available under MATERIALS AND METHODS aCC-BY 4.0 International license.

CeMEE genetic structure

We assessed accuracy and appropriate variant filtering thresh- 347 olds by genotyping a set of 784 markers, uniformly distributed 348 across the six chromosomes according to the genetic distances 349 of Rockman and Kruglyak (2009), in 182 RILs with the iPlex 350 Sequenom MALDI-TOF platform (Bradić et al. 2011). Sequenom 351 data can be found in Table S2. We fitted a linear model with ${ }_{352}$ counts of Illumina/Sequenom concordant cases as the response ${ }_{353}$ variable, and all founder variant quality metrics together with 354 the number of missing or heterozygous calls in the founders, 355 the number of zero-coverage or potentially heterozygous sites 356 (with at least a single Illumina read for each genotype), variant ${ }_{357}$ nucleotide identity, and reference nucleotide and dinucleotide 358 identity as explanatory variables. Concordance across sequenc- 359 ing platforms was $96.9 \%$ after ( $93.7 \%$ before) final filtering, and 360 we retained 388,201 diallelic SNPs as founder markers. We es- 361 timated residual heterozygosity for 25 A140 lines sequenced 362 to $>20 \mathrm{X}$ coverage (single sample calls using GATK 3.3-0 Hap- 363 lotypeCaller, variant filtration settings $\mathrm{MQ}<50.0$ । । $\mathrm{DP}<5364$ | | MQRankSum $<-12.5$ | | SOR $>6$ | | FS $>60.0$ || Read- 365 PosRankSum $<-8.0||$ QD $<10.0|| \mathrm{DP}>$ mean $\times 3$ ). Mean 366 heterozygosity at founder sites is $0.095 \%$ (standard deviation 367 $0.042 \%$, range $0.033-0.18 \%$ ).

After removal of RILs sharing greater than the mean pairwise ${ }^{369}$ identity +5 standard deviations $(84.8 \%$, excluding monoecious 370 lines), we retained 178 A140 RILs, 118 CA50 RILs (from three ${ }^{371}$ replicate populations), 127 GA50 RILs (three replicates), and ${ }^{372}$ 79 GT50 RILs (two replicates). The 98 GM50 RILs (two repli- 373 cates) are highly related on average and group together into a 374 small number of "isotypes". To prevent introduction of strong ${ }^{375}$ structure, we discard all but five below the above panel-wide ${ }^{376}$ pairwise identity threshold for the purposes of trait mapping. In ${ }^{377}$ total, the CeMEE comprises 507 RILs from five sub-panels, with 378 352,583 of the founder markers segregating within it (File S3).

\section{CeMEE genetic structure}

Differentiation from natural isolates and founders We compared similarity within and between the CeMEE RILs and 152 sequenced wild-isolates from the CeNDR panel (release 20160408). The distributions for all pairwise genotype and haplotype (\% identity at $0.33 \mathrm{cM}$ scale in $F_{2}$ map distance) distances are plotted in Figure 2, for 256,535 shared diallelic sites with no missing or heterozygous calls.

Linkage disequilibrium $\left(r^{2}\right)$ was computed for founders and ${ }_{391}^{390}$ CeMEE RILs at the same set of sites (MAF $>1 / 16,<5 \%$ ambiguous imputed RIL genotypes and $\leq 1$ heterozygous/missing 392 founder genotypes, then downsampled by 10 for computational 393 tractability), and plotted against genetic distances (obtained by 394 linear interpolation from the N2/CB4856 map, scaled to $F_{2}$ dis- 395 tances (Rockman and Kruglyak 2009). To assess the extent of 396 subtle, long-range linkage disequilibrium in the form of inter- 397 chromosomal structure, we compared $r^{2}$ among chromosomes 398 to a null distribution generated by permutation $(n=5000)$. In 399 each permutation, filtered RIL genotypes (pruned of strong local 400 linkage $r^{2}<0.98$, no ambiguous calls) were randomly down- 401 sampled to equal size across chromosomes, split by chromosome, 402 then shuffled within each sub-panel before taking the mean cor- 403 relation across chromosomes (or omitting all single and pairwise 404 chromosome combinations) as test statistic. The effect of local 405 LD pruning is to reduce the weighting of large regions in strong 406 linkage in order to better assay weak interactions across the 407 remainder of the genome.
Reconstruction of ancestral haplotypes and genetic map expansion For each RIL, founder haplotypes were inferred with the RABBIT HMM framework implemented in Mathematica (Zheng et al. 2015), conditioning on the recombination frequencies observed for the N2 $\times$ CB4856 RILs (scaled to $F_{2}$ map length) (Rockman and Kruglyak 2009). Realized map expansion was estimated by maximum likelihood for each chromosome, before full marginal reconstruction of each chromosome (explicitly modeling recombination on the $\mathrm{X}$ and autosomes) using posterior decoding under the fully dependent homolog model (depModel). Under this model, appropriate for fully inbred diploids, chromosome homologs are assumed to have identical ancestral origins (prior identity by descent probability $f=1$ ), and the recombination junction density (transition probability) is given by the estimated map expansion $(R a)$ and genotyping error rates (set to $5 \times 10^{-5}$ for founders and $5 \times 10^{-3}$ for RILs based on likelihood from a parameter sweep). Sites called as heterozygous or missing in the founders, or unresolved to $[0,1]$ by the genotype imputation HMM were set to NA before reconstruction. For reconstruction summaries, haplotype posterior probabilities were filtered to $>0.2$, and haplotype lengths and breakpoints were estimated from run lengths of marker assignments, taking the single best haplotype (if present), maintaining haplotype identity (if multiple assignments of equal probability), or the first among equals otherwise.

To test reconstruction accuracy as a function of haplotype length, we performed simulations of a pedigree varying only the number of generations of random mating. Starting from a single population representing all founders $(\mathrm{N}=1000$, corresponding to the expected $N_{e}$ during experimental evolution), mating occurred at random with equal contribution to the next generation. Recombination between homologous chromosomes occurred at a rate of $50 \mathrm{cM}$, with full crossover interference, and the probability of meiotic crossover based on distances between marker pairs obtained by linear interpolation of genetic positions (Rockman and Kruglyak 2009). For each chromosome, 10 simulations were run sampling at 10, 25, 50, 100 and 150 generations, and haplotype reconstruction was carried out as above. Maximum likelihood estimates of realized map expansion for simulations were used to calibrate a model for prediction of realized number of generations in the RILs by chromosome. A 2nd degree polynomial regression of $R a$ on the known number of generations was significantly preferred over a linear fit by likelihood ratio test, given significant underestimation as pedigree length increased (approaching $10 \%$ at $G_{150}$ ).

Population stratification Population stratification was assessed using (1) principal component decomposition, giving a uni- or bivariate view of the importance of genetic structure associated with CeMEE sub-panels, and (2) by supervised and unsupervised discriminant analysis of principal components (DAPC; Jombart et al. (2010)), giving an estimate of the fraction of principal component variance that best predicts sub-panel structure, and an inference of population structure without regard to subpanel identities. In all cases decomposition was of scaled and centered genotypes pruned of strong local LD $\left(r^{2}<0.98\right)$, giving all markers equal weight (and therefore more weight to low frequency alleles).

Of the first 50 principal components, 10 are significantly associated with sub-panel identity (i.e., evolutionary history) by ANOVA ( $p<0.05$ after Bonferroni correction), accounting for just $3.9 \%$ of the variance in sum. Seven of the top 10 PCs are significant, though others up to PC 38 are also associated, showing 
bioRxiv preprint doi: https://doi.org/10.1101/120865; this version posted March 27, 2017. The copyright holder for this preprint (which was not certified by peer review) is the author/funder, who has granted bioRxiv a license to display the preprint in perpetuity. It is made available under Heritability and phenotype prediction aCC-BY 4.0 International license.

that multiple sources of structure contribute to the major axes of 470 variation. Fitting all pairs among the the top 50, two pairs (7 and 471 19, 13 and 14) are significant (again at a conservative Bonferroni 472 adjusted threshold), resolving the GT50 RILs as most distinct. $\quad 473$

For DAPC (R package adegenet, Jombart (2008)), we used 474 100 rounds of cross-validation to determine the number of prin- 475 cipal components required to achieve optimal group assignment 476 accuracy (the mean of per-group correct assignments). This 477 value (40 PCs) was then used to infer groups by unsupervised 478 $k$-means clustering (default settings of 10 starts, $10^{5}$ iterations), 479 with $k$ selected on the Bayesian Information Criterion (BIC). Cor- 480 respondence of inferred groups with known groups was tested 481 by permutation. Given the contingency table $C$, where $C_{i, j}$ repre- 482 sents the number of lines known to be in sub-panel $i$ and inferred ${ }^{483}$ to be in cluster $j$, the inferred values for each cluster (js) were ${ }^{484}$ shuffled among known groups (is) 10,000 times, with the sum of 485 the variance among known groups taken as a summary statistic 486 (high values reflecting significant overlap between inferred and known groups).

\section{Phenotyping}

Fertility In the experimental evolution scheme under which the 49 CeMEE RILs were generated, a hermaphrodite's contribution 492 to the next generation is the number of viable embryos that ${ }_{493}$ survive bleaching (laid, but unhatched, or held in utero) that 494 subsequently hatch to L1 larvae $24 \mathrm{~h}$ later. We treat this pheno- 495 type as fertility, and measured it for individual worms of 230496 RILs. Each line was thawed and maintained for two generations 497 under standard conditions (Stiernagle 2006; Teotónio et al. 2012; 498 Theologidis et al. 2014), bleached to kill adults, then embryos 499 were allowed to hatch and synchronize as L1 larvae. L1s were 500 then moved to fresh plates seeded with E. coli and allowed to 501 develop for 48 hours. Single L3-L4 staged hermaphrodite lar- 502 vae were then placed into each well of 96-well plates using a ${ }_{503}$ micropipette and stereomicroscope. Plate wells contained NGM- 504 lite $+100 \mu \mathrm{g} / \mathrm{ml}$ ampicillin, previously inoculated with $1 \mu \mathrm{l}$ of 505 an overnight culture of $E$. coli (HT115) and stored until usage at 506 4C (maximum 2 weeks before use). After transfer, plates were 507 covered with Parafilm to prevent cross-contamination and incu- 508 bated at 20C and $80 \%$ relative humidity (RH) until the following 509 day. Embryos were extracted by adding bleach solution to wells 510 $(1 \mathrm{M} \mathrm{KOH}, 5 \% \mathrm{NaClO} 1: 1 \mathrm{v} / \mathrm{v}$ in M9 buffer) for 5 minutes, then 511 $200 \mu \mathrm{l}$ of the extract was removed and rinsed 3 times in M9 buffer 512 by centrifugation. The M9 suspension $(200 \mu \mathrm{l})$ was then trans- 513 ferred to another 96-well plate containing $120 \mu \mathrm{l}$ of M9 per well. ${ }_{514}$ Plates were incubated overnight (as above), then centrifuged 515 for $1 \mathrm{~min}$ at 1800rpm to sediment any swimming larvae before 516 imaging at $4 \mathrm{pixel} / \mu \mathrm{m}^{2}$ with a Nikon Eclipse TE2000-S inverted 517 microscope. ImageJ was then used to manually count the num- 518 ber of live (moving) L1s in each well. During assay setup and 519 image analysis wells were censored where: bacteria were absent; 520 hermaphrodites were absent or dead at the time of bleach; males 521 had been inadvertently picked; more than 1 adult was present; 522 or hermaphrodites had not been killed upon bleaching. Except ${ }_{523}$ for density between the L4 stage until reproduction, all assay 524 conditions were the same as those used during experimental evolution. Fertility measurements do not include potential sur- 525 vival differences between the L1 stage until reproduction, but we ${ }_{526}$ nonetheless take it as a surrogate for fitness (Chelo et al. 2013). ${ }_{527}$

Two independent plates within a single thaw were set-up ${ }_{528}$ for most RILs ( 1 plate for six lines, maximum=4, mean=2.0), 529 which we consider as replicates for estimation of repeatability 530 (see below). In total the median number of measurements per line was 43 (range 4-84). Highly replicated data for the reference strain N2 were also included for modeling purposes (404 observations across 17 plates, spanning 9 of 47 independent thaws). Wells with no offspring were observed for $4 \%$ of N2 data (and $2.9 \%$ of all RIL data). These are likely to be due to technical artifact, such as injury or incorrect staging, and were excluded before modeling. Mapping values were the Box-Cox transformed line coefficients from a Poisson generalized linear model with fixed effects of plate row, column and edge (exterior rows and columns), and the count of offspring per worm as response variable. Three outliers with coefficients $>3$ standard deviations below the mean were excluded, leaving data for 227 RILs (File S4). Data come from RILs of three sub-panels (170 A6140, 45 GA50, 12 GT50), which explains 4\% of trait variation (GA50 RILs have higher mean fertility than the A6140, regression coefficient $=0.43, p=0.01$; see Figure S1).

Adult hermaphrodite body size 412 RILs were thawed and maintained for two generations under standard conditions. On the third generation, 1000 synchronized L1 larvae were moved to NGM-lite plates $(25 \mathrm{mM} \mathrm{NaCl})$ where they developed and matured for 3 days. Image data was acquired at the usual time of reproduction (as during experimental evolution) and analysed with the Multi-Worm Tracker (Swierczek et al. 2011), using a Dalsa Falcon 4M30 CCD camera and Schott backlight A08926. Tracking was performed for 25 minutes with default parameters, and particle (worm) contours extracted (on average, 300 particles obtained every $0.5 \mathrm{~s}$ ). Raw values from each plate were calculated from track segments of length 40-41s taken at 80s intervals, ultimately estimating the area of an individual as the grand mean of the per-segment estimates (accounting for temporal autocorrelation within a time-series, analysis not shown).

Assays were carried out in two lab locations over several years, while recording the relative humidity and temperature at the time of assay. Mapping values are the Box-Cox transformed line coefficients from a linear model incorporating fixed effects of year, nested within location, and humidity and temperature, nested within location. Data come from a mean of 2.1 (maximum 4) independent thaw blocks for each RIL, for 410 RILs after excluding 2 outliers $>3$ standard deviations below the mean, with a median of 447 measurements per RIL and block (range 109-1013; File S5). Data for the reference strain N2 were also included in the model (1664 observations from two plates). Data come from RILs of three sub-panels (165 A6140, 118 CA50, 127 GA50), which explains $17 \%$ of trait variation (GA50 RILs are much larger than the A6140, regression coefficient $=0.94, p<$ $10^{-16}$; see Figure S1). This difference is not obviously associated with technical covariates, since data acquisition for A140 RILs and GA50 RILs was distributed similarly with respect to location and time.

Fertility and body size are moderately correlated (Figure S1; see also Poullet et al. (2016)), justifying the latter being considered a fitness-proximal trait (Spearman's $\rho=0.354, p=$ $2.336 \times 10^{-7}$ for mapping coefficients, for 202 lines with data for both traits).

\section{Heritability and phenotype prediction}

Repeatability Repeatability was estimated from ANOVA of the line replicate means for each trait as $R=\sigma_{a}^{2} /\left(\sigma_{a}^{2}+\sigma_{e}^{2}\right)$, where $\sigma_{a}^{2}=$ (mean square among lines - mean square error) $/ n_{0}$, and $n_{0}$ is a coefficient correcting for varying number of observations (1-4 plate means) per line (Lessells and Boag 1987; Sokal and 
Rohlf 1995). Assuming equal variance and equal proportions of environmental and genetic variance among replicates, $R$ represents on upper bound on broad-sense heritability (Falconer 1981; Hayes and Jenkins 1997). Fertility data were square root transformed to decouple the mean and variance.

Assumptions In inbred, isogenic, lines, broad-sense heritability can also be estimated by linear mixed effect model from the covariance between genetic and phenotypic variances. The measurement of genetic similarity is, however, subject to a number of assumptions and is (almost) always, at best, an approximation (Speed and Balding 2015).

A first assumption is that all markers are the causal alleles of phenotypic variation. It is unavoidable, however, that markers tag the (unknown) causal alleles to different degrees due to variable linkage disequilibrium. A second, usually implicit, assumption in calculating genetic similarity is the weight given to markers as a function of allele frequency. Equal marker weights have commonly been used in animal breeding research, while greater weight has typically been given to rare alleles in human research, which has some support under scenarios of both selection and neutrality (Pritchard 2002). A third assumption, related to the first two, is the relationship between LD and causal variation. If the relationship is positive - causal variants being enriched in regions of high LD - then heritability estimated from all markers will be upwardly biased, since the signal from causal variation contributes disproportionately to genetic similarity (Speed et al. 2012).

The use of whole genome sequencing largely addresses the first assumption, given (as here) very high marker density and an accurate reference genome, although in the absence of full de novo genomes from long-read data for each individual, the contribution of large scale copy-number and structural variation, and new mutation, will remain obscure. To account for the second and third assumptions, we used LDAK (v5.0) to explicitly account for LD in the CeMEE (decay half-life $=200 \mathrm{~Kb}$, min-cor $=$ 0.005 , min-obs $=0.95)($ Speed et al. 2012). Heritability estimates were not sensitive to variation in the decay parameter over a 10-fold range or to the measurement unit (physical or genetic), although model likelihoods were non-significantly better for physical distance. Across the set of 507 RILs, 88,508 segregating markers were used after local LD-based pruning $\left(r^{2}<0.98\right)$ and, of these, 22,984 markers received non-zero weights. LDweighting can magnify the effects of genotyping errors. We excluded 17,740 markers with particularly low local LD (mean $r^{2}$ over a 20 marker window $<0.3$, or the ratio of mean $r^{2}$ to that of the window mean $<0.3$ ). Heritability estimates were largely unchanged (within the reported intervals), as were our general conclusions on variance components and model performance.

Modeling Model fit was assessed by phenotype predictions from leave-one-out cross validation, calculating the genomic best linear unbiased prediction (GBLUP; Meuwissen et al. (2001); VanRaden (2008); Yang et al. (2010)) for each RIL and returning the squared correlation coefficient $\left(r^{2}\right)$ between observed and predicted trait values. To avoid bias associated with sample size all models were unconstrained (non-error variance components were allowed to vary outside 0-1 during convergence) unless otherwise noted, which generally gave better fit for multicomponent models.

Given $m$ SNPs, genetic similarity is calculated by first scaling $S$, the $n \times m$ matrix of mean centered genotypes, where $S_{i, j}$ is the number of minor alleles carried by line $i$ at marker $j$ and frequency $f$, to give $X$ :

$$
X_{i, j}=\left(S_{i, j}-2 f_{j}\right) \times\left(2 f_{j}\left(1-f_{j}\right)\right)^{\frac{\alpha}{2}} ;
$$

The additive genomic similarity matrix (GSM) $\mathbf{A}$ is then $\mathbf{X} \mathbf{X}^{T} / m$. Here $\alpha$ scales the relationship between allele frequency and effect size (Speed et al. 2012). $\alpha=-1$ corresponds to the assumption of equal variance explained per marker (an inverse relationship of effect size and allele frequency), while common alleles are given greater weight at $\alpha>0$. We tested $\alpha \in[-1.5,-1,-0.5,0,0.5,1]$ and report results that maximized prediction accuracy. With $Y$ the scaled and centered vector of $n$ phenotype values, the additive model fit for estimating genomic heritability $h^{2}$ is then

$$
\begin{aligned}
& Y=\sum^{m} \beta A+e, \\
& \quad \text { with } \beta \sim \mathcal{N}\left(0, \sigma_{g}^{2}\right), e \sim \mathcal{N}\left(0, \sigma_{e}^{2}\right)
\end{aligned}
$$

where $\beta$ represents random SNP effects capturing genetic variance $\sigma_{g}^{2}, e$ is the residual error capturing environmental variance $\sigma_{e}^{2}$. Given $Y$ and $\mathbf{A}$, heritability can be estimated from restricted/residual maximum likelihood (REML) estimates of genetic and residual variance as $h^{2}=\sigma_{g}^{2} /\left(\sigma_{g}^{2}+\sigma_{e}^{2}\right)$. Note that we use the terms $h^{2}$ and genomic heritability interchangeably here for convenience, although in some cases the former includes non-additive covariances. We assume RILs are fully inbred, and so dominance variance does not contribute to heritability.

The existence of near-discrete recombination rate domains across chromosomes has lead to characteristic biases in nucleotide variation, correlated with gene density and function (Cutter et al. 2009). Similarly, recent selective sweeps, coupled with the low effective outcrossing rate in C. elegans, have lead to a markedly unequal distribution of variation across chromosomes (Andersen et al. 2012; Rockman et al. 2010). This variability in mutational effect, along with variable LD in the RILs, is not captured by aggregate genome-wide similarity with equal marker weighting (Speed et al. 2012; Goddard et al. 2016). We therefore first tested genetic similarity by explicitly modeling observed LD (Speed et al. 2012), with markers weighted by the amount of genetic variation they tag along chromosomes, and by their allele frequency (see above). Given $m$ weights reflecting the amount of linked genetic variation tagged by each marker, $w_{i}, \ldots, w_{m}$, the variance covariances for the basic model become:

$$
\beta \sim \mathcal{N}\left(0, w \sigma_{g}^{2} / W\right)
$$

where $W$ is a normalizing constant. Second, we jointly measured the variance explained by individual chromosomes (and by recombination rate domains within each chromosome), which can further improve the precision of heritability estimation if causal variants are not uniformly distributed by allowing variance to vary among partitions. Third, we tested epistatic as well as additive genetic similarity with (1) the entrywise (Hadamard) product of additive GSMs, giving the probability of allele pair sharing (Henderson 1985; Jiang and Reif 2015), (2) higher exponents up to fourth order interactions and (3) haplotype-based similarity at multi-gene scale. Additional similarity components (additive or otherwise) are added as random effects to the above model to obtain independent estimation of variance components:

$$
\begin{aligned}
Y & =\sum^{m} \beta A_{1}+\ldots+\beta_{n} A_{n}+e, \\
\beta_{1} & \sim \mathcal{N}\left(w \sigma_{g 1}^{2} A_{1} / W\right), \\
\beta_{n} & \sim \mathcal{N}\left(w \sigma_{g n}^{2} A_{n} / W\right), \\
e & \sim \mathcal{N}\left(0, \sigma_{e}^{2}\right)
\end{aligned}
$$


bioRxiv preprint doi: https://doi.org/10.1101/120865; this version posted March 27, 2017. The copyright holder for this preprint (which was not certified by peer review) is the author/funder, who has granted bioRxiv a license to display the preprint in perpetuity. It is made available under Data Availability aCC-BY 4.0 International license.

Haplotype similarity was calculated as the proportion of identi- 653 cal sites among lines at 0.033 and $0.067 \mathrm{cM}$ scales (corresponding ${ }_{654}$ to means of approximately 5 and $10 \mathrm{~Kb}$ non-overlapping block 655 sizes, or one and two genes), using either the diallelic markers 656 only, or all called SNPs and indels. In the latter case, variants 657 were imputed from reconstructed haplotypes if the most likely 658 haplotypes of flanking markers were in agreement.

\section{GWAS}

1-dimensional tests For single trait, single marker association, 662 we fitted linear mixed models using the Python package LIMIX ${ }^{663}$ (https://github.com/PMBio/limix):

$$
Y=\beta X+g+e, \text { with } g \sim \mathcal{N}\left(0, \sigma_{g}^{2} A\right), e \sim \mathcal{N}\left(0, \sigma_{e}^{2}\right)
$$

where $X$ is the matrix of fixed effects (the SNP genotype of ${ }_{668}$ interest) and $\beta$ is the effect on phenotypic variation that is esti- ${ }_{669}$ mated. $g$ are the random effects describing genetic covariances 670 (as above) accounting for non-independence among tests due 671 to an assumed polygenic contribution to phenotype, with A the 672 $n \times n$ genetic similarity matrix from the most predictive additive 673 fit found for each trait above, and $e$ is the error term.

To test the mapping resolution and power of the CeMEE 675 panel, we carried out GWAS according to the model above for 676 simulated phenotypes. We modeled a single focal additive locus 677 (with $h^{2}$ from 1 to $30 \%$ ) and a background polygenic component ${ }_{678}$ of equal variance (with scenarios of 10, 100 or 1000 loci), selected 679 at random from SNPs with MAF > 0.05, and with genetic and 680 environmental effect sizes drawn independently from the stan- 681 dard normal distribution. GWAS was carried out 1000 times for each scenario, controlling for relatedness with LD-weighted additive genetic similarity $(\alpha=-0.5)$. Power was estimated from a binomial generalized linear model considering all three polygenic scenarios together. Recall, the proportion of true positives passing significance, was assessed after masking a $1 \mathrm{cM}$ window around the focal SNP. 2-LOD drop intervals around the focal locus were calculated from similarly powered mark- 686 ers with $\geqslant$ MAF, with $p$-values converted to LOD scores as $\left.\chi^{2} / 2 \times \log (2) / \log _{10}(2)\right)$.

For simulated traits all 507 lines and 262,218 markers (MAF > 0.05 ) were used for GWAS. For body size GWAS 410 lines and 254,174 markers were used, and 227 lines and 254,240 markers were used for fertility. Significance thresholds were established by permutation, with phenotypes generated by permuting phenotype residuals, given the estimated relatedness among lines (A), using the R package mvnpermute (Abney 2015). Significance level $\alpha$ is the corresponding percentile of the minimum $p$-values from 1000 permutations.

Given the correlation between traits (see above), we also tested a model for each trait on phenotype residuals after linear regression on the other, and a multi-trait model fitting effects common or specific to a trait. No markers passed significance in any case (analysis not shown).

2-dimensional tests We tested for additive-by-additive epistasis on the assumption of complete homozygosity. We first reduced the search space by local LD pruning $\left(r^{2}<0.5\right)$, requiring MAF $>0.05$, the presence of all four two-locus homozygote classes at a frequency of $\geqslant 3$, with $\leqslant 5$ missing or ambiguous imputed genotypes (which were excluded from analysis). This gave a 70 total of 19,913,422 tests for fertility (both inter- and intrachromo- 708 somal) and 28,138,090 for size, across 9,628 and 10,329 markers 709 respectively. We tested for main and interaction additive effects 710 for all marker pairs by ANOVA, taking as summary statistics the $F$-statistic for genotype interaction (2D tests), and also the sum of interaction scores for each marker (2D sum tests) above each of three thresholds $(F>0,8,16$, the latter corresponding roughly to the most significant single marker associations seen for both traits). All statistics were calculated separately for inter- and intrachromosomal tests. 2D sum tests are testing for excess weak to moderate interactions due to polygenic epistasis.

For computational tractability, tests were run in parallel on two chromosomes at a time. Null permutation thresholds were generated by shuffling phenotypes (using mvnpermute as above to ensure exchangeability in the presence of polygenicity or structure). 2D test thresholds were calculated for each chromosome separately from at least 2000 permutations each and differed little across chromosomes $\left(\alpha=10 \%, 2.86-1.16 \times 10^{-7}\right.$ for fertility, $1.86 \times 10^{-7}-7.2 \times 10^{-8}$ for size). Inter- and intrachromosomal thresholds were calculated separately, but the reported interactions do not change if we pool both classes (or all chromosomes). 2D sum test thresholds were calculated separately for each chromosome pair and class (inter- and intrachromosomal).

We initially ignored relatedness for 2D testing, then fit linear mixed effect models as above with genetic covariance $\mathbf{A}$ for candidate interactions (R package hglm; Shen et al. (2014)). For size, the two candidate interactions all decreased slightly in significance (to a maximum $p$-value of $7.8 \times 10^{-7}$ ), while significance increased for all four fertility interactions. The amount of phenotypic variance explained by candidates for each trait was estimated by ANOVA, jointly fitting all main and two-locus interactions.

\section{Data Availability}

Sequence data are available from NCBI SRA under accession XXXXX. All data and methods scripts are archived in Dryad.org doi: XXX. RILs are available from the authors.

\section{Results and Discussion}

\section{CeMEE differentiation from natural populations}

The CeMEE panel of recombinant inbred lines draws variation from sixteen founders, and shuffles the diversity they contain through more than 150 generations at moderate population sizes and predominant outcrossing. The wild founders used to create the panel together carry approximately $25 \%$ of single nucleotide variants known to segregate in the global C. elegans population (CeNDR; Caenorhabditis elegans Natural Diversity Resource; Cook et al. (2017)). They vary, however, in distance to the N2 reference strain, with the Hawaiian CB4856 and German MY16 isolates together contributing over half of all markers, while the Californian CB4507 is closely related to N2 (Figure S3). Comparison of pairwise genetic distances in the CeMEE and 152 sequenced wild isolates (including a small number of more recently isolated, highly divergent lines) illustrates the extent of novelty generated by the multiparental cross (Figure 2). The CeMEE RILs occupy a substantial sub-space of the CeNDR genotypic diversity (Figure 2A), without the extensive haplotype sharing among wild-isolates and with the creation of many new multigenic haplotypes (Figure 2B).

\section{CeMEE differentiation from parental founders}

Since C. elegans natural isolates suffer from outbreeding depression (?Gimond et al. 2013), the mixing phase is expected to generate high variance in fitness which, channeled through 
bioRxiv preprint doi: https://doi.org/10.1101/120865; this version posted March 27, 2017. The copyright holder for this preprint (which was not certified by peer review) is the author/funder, who has granted bioRxiv a license to display the preprint in perpetuity. It is made available under

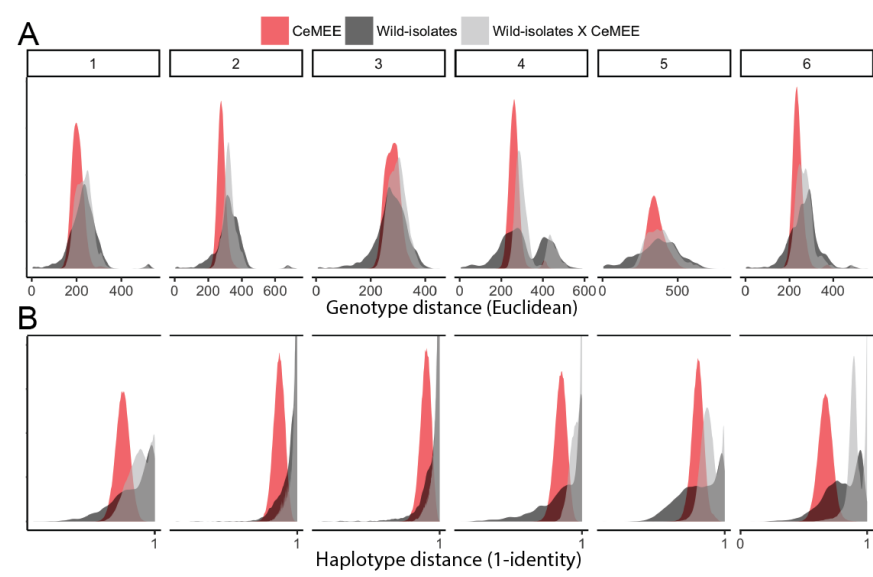

Figure 2 Similarity among CeMEE RILs and 152 sequenced wild-isolates (Caenorhabditis elegans Natural Diversity Resource) at 256,535 shared diallelic sites. The distribution of pairwise genotype (A) and haplotype (B) distances, within and between CeMEE RILs and CeNDR wild-isolates, by chromosome. Haplotype distances are $1-\%$ identity at $0.1 \mathrm{cM}$ scale. Note that chromosomes 2-4 all show a marked excess in haplotype dissimilarity between CeMEE RILs and CeNDR wildisolates, and the density is truncated by a factor of four for visibility.

of alleles more generally. These effects are expected to depend on reproductive mode and selection (Charlesworth and Wright 2001; Morran et al. 2009; Chelo and Teotónio 2013; Chelo et al. 2014; Kamran-Disfani and Agrawal 2014) and will be addressed in future work.
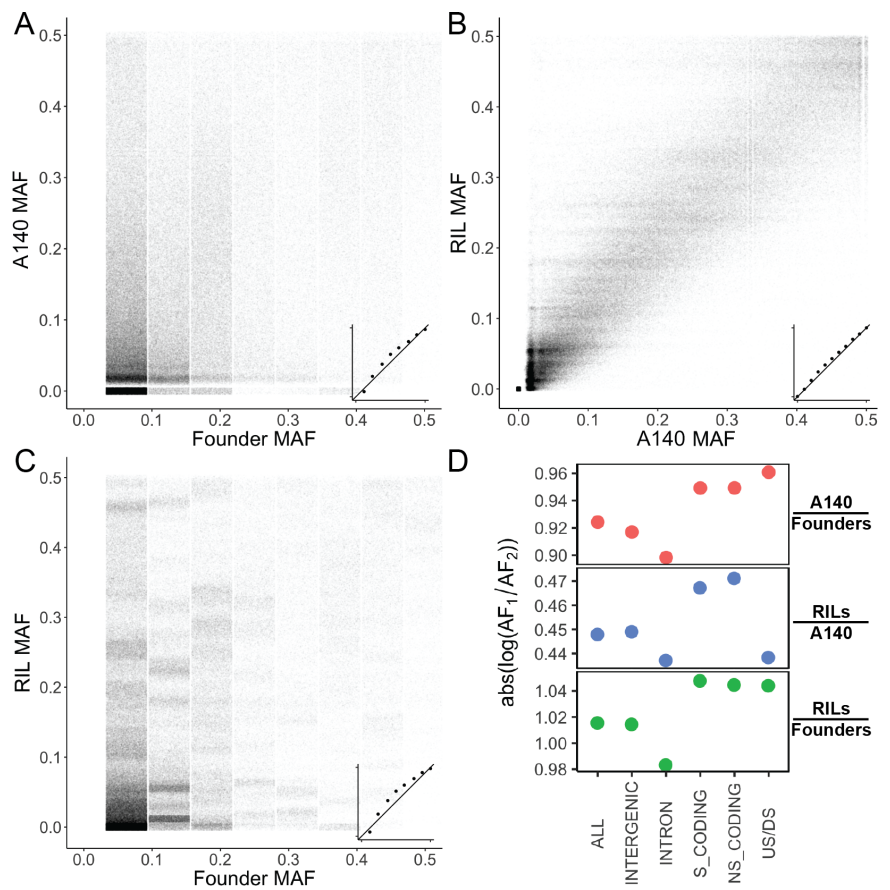

$D$

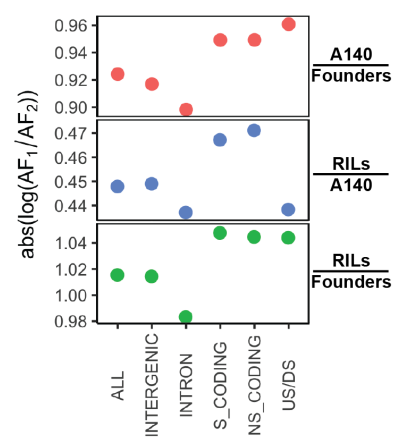

Figure 3 Minor allele frequency between founders and the outbred A140 population (A), A140 and RILs (inbreeding only for the A140 RILs, further adaptation then inbreeding for G50 RILs; B), and founders against all RILs (C). Insets show frequency quantiles. D. Change in allele frequency (absolute log ratios) for the same contrasts by functional class: intronic, synonymous and non-synonymous, putative regulatory variation (US/DS; $\leq 200 b p$ from an annotated transcript or N2 pseudogene), or intergenic (none of the above). Points are mean values (diameter exceeds the standard errors).

Local linkage disequilibrium, while non-uniform among chromosomes, decays relatively rapidly on average, approaching background levels by $0.5 \mathrm{cM}\left(\mathrm{F}_{2}\right.$ map scale) on average (Figure 4 and Figure S2). Disequilibrium between pairs of loci on different chromosomes is, as expected, very weak $(0.99,0.95$ quantiles $=0.538,0.051$ within chromosomes versus $0.037,0.022$ across chromosomes), with the prominent exception of a single pair of loci on chromosomes II and III $\left(r^{2}>0.5\right.$ between II:2,284,322; tagging an intact MARINER5 transposon (WBTransposon00000128) that harbors an expressed miRNA in the N2 reference, and III:1,354,894-1,425,217; a broad region of mostly unannotated genes, against maximum interchromosomal values for all other pairs $\left.r^{2} \leqslant 0.27\right)$. Alleles in repulsion phase are rare across these regions $\left(p<10^{-70}\right.$, Fisher Exact Test), absent in the founders, and present in only 1 of 124 wild isolates surveyed with unambiguous variant calls in these regions (Caenorhabditis elegans Natural Diversity Resource). This suggests the presence of at least one two-locus incompatibility exposed by inbreeding or, perhaps more likely given the uncertainties of referencebased genotyping, a transposon-mediated II-III transposition polymorphism among founders. Three founders contribute the 

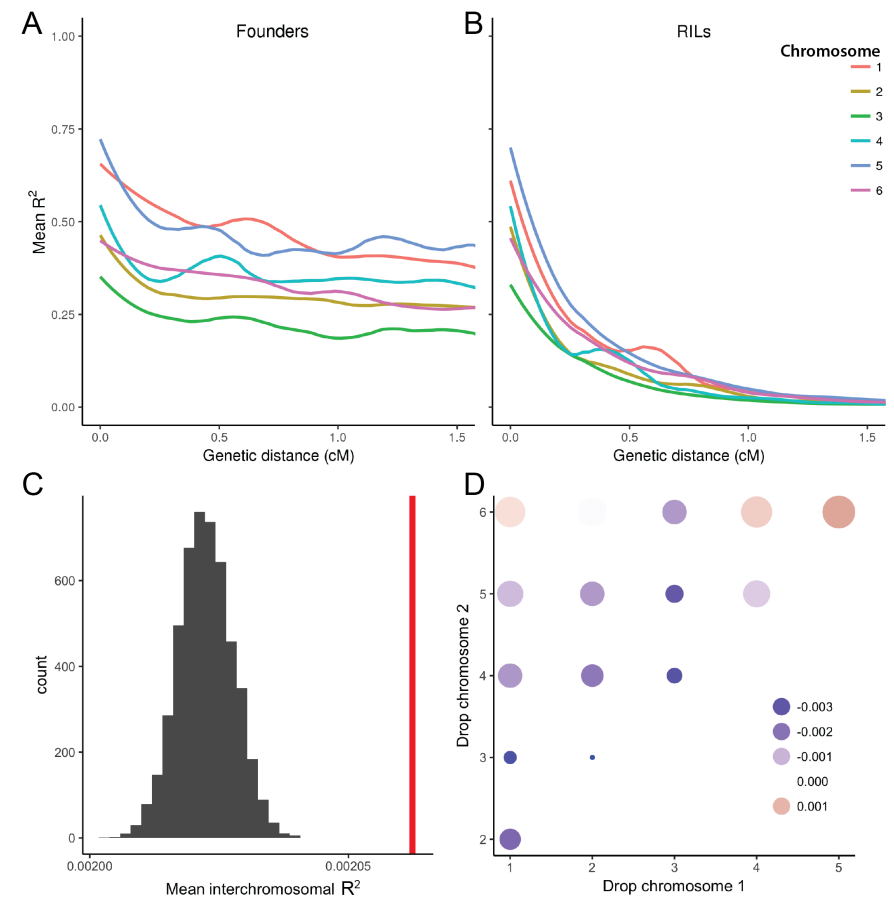

Figure 4 Linkage disequilibrium in founders (A) and all CeMEE RILs (B; $F_{2}$ genetic map distance, LOESS fit to mean $\left.r^{2}\right)$. C. Interchromosomal structure is weak but significant. Observed mean $r^{2}$ across all chromosomes (red vertical bar) plotted against the null distribution from permutations randomizing lines across chromosomes (within sub-panels to exclude effects of population structure). D. Permutations dropping pairs of chromosomes implicate $\mathrm{X}$-autosome interactions. Point size and color is scaled by enrichment over the null distribution (95\% percentile), relative to the genome-wide mean value.

\section{Founder haplotype blocks and genetic map expansion}

The CeMEE panel is highly recombined and any simple, lar effect incompatibilities between founders are likely to have been 851 purged. For example, a haplotype containing peel-1 and zeel-1, a 852 known incompatibility locus that segregates among the founders 853 on the left arm of chromosome I (Seidel et al. 2008, 2011), is fixed 854 in the RILs (Figure 5a). Cases such as this are best appreciated 855 when the mosaic of founder haplotypes across the genome is 856 inferred.
For each CeMEE RIL, founder haplotypes across the genome were reconstructed with the multiparent HMM framework RABBIT (Zheng et al. 2015), assigning $96.9 \%$ of markers to a single founder haplotype at posterior probability $>0.2(84.2 \%>0.5$; median value across lines. Haplotype sharing in the 16 founders means that unambiguous assignment to a single founder is not always possible). For illustration purposes, a summary of reconstructed haplotypes for the A140 RILs on chromosomes I, IV and $X$ are shown in Figure 5, at both physical and genetic scales to make the differences between these units plain. The observed recombination landscapes generally recapitulate those inferred from the N2/CB4856 cross (Rockman and Kruglyak 2009; Kaur and Rockman 2014; Bernstein and Rockman 2016), with recombination rate high in chromosome arms and low in centers. With the additional map expansion gained here (see below), we note that suppression of recombination is clearly strong, but not complete, within subtelomeric regions (see, for example, the exceptionally large right tip of chromosome $\mathrm{X}$, spanning almost $2 \mathrm{Mb}$, in Figure $5 \mathrm{c}$ ).

Founder haplotype diversity among all CeMEE RILs remains high: the median number of founder haplotypes across reconstructed intervals is 12 (posterior probability $>0.5$, haplotypes observed in $>1$ RIL). Contributions clearly vary from equality, with lines most divergent from the reference (CB4856 and MY16) overrepresented and lines more similar to the reference underrepresented (with the exception of the large region of chromosome $\mathrm{X}$, spanning $n p r-1$, which is largely fixed for N2/CB4507 alleles (Figure 5c). To establish if these biases are merely technical, and establish expectations for reconstruction resolution in the presence of haplotype redundancy, we simulated genomes of varying pedigree length (up to 150 generations). As expected, reconstruction was hampered by increasing recombination, and by ambiguity between similar founders (Figure S3). Bias toward divergent haplotypes was not observed in the reconstruction simulations, however, suggesting the overrepresentation of CB4856 and MY16 may be due to selection, notably for long haplotypes across the central domain of chromosome IV (Figure 5b). Reconstruction completeness for the A140 RILs is generally in line with expectations for a pedigree of 150 generations. Clear exceptions are chromosome IV, where we recover more than expected under random sampling, and chromosome V, where we recover less. Haplotype lengths from simulated reconstructions showed we progressively underestimate recombination breakpoints due to imperfect resolution of small haplotypes (Figure S3).

The relationship between known generation and estimated realized map expansion from reconstruction simulations allows prediction of the number of effective generations of outcrossing within the CeMEE panel. Across the five sub-panels, mean autosomal generation ranges from 227 (GM monoecious RILs) to 284 (CA androdioecious lines), with a weighted average of 260 for the CeMEE as a whole (Figure S4). Estimated genetic map expansion is variable across chromosomes: IV appears to be exceptionally recombinant in all sub-panels with expansion more than twice that of chromosomes I-III, due largely to a high frequency, highly structured haplotype on the far right arm and tip (Figure 5b). This region spans one of the two large C. elegans piRNA clusters (Ruby et al. 2006), which encodes more than 15,000 piRNA transcripts, interspersed with active transposons and protein coding genes. Several trivial explanations for the unusual apparent expansion, such as elevated genotyping error rate or founder haplotype ambiguity, or distortions in the N2/CB4856 genetic map use to condition reconstruction 
bioRxiv preprint doi: https://doi.org/10.1101/120865; this version posted March 27, 2017. The copyright holder for this preprint (which was not certified by peer review) is the author/funder, who has granted bioRxiv a license to display the preprint in perpetuity. It is made available under RESULTS AND DISCUSSION aCC-BY 4.0 International license.

probabilities, are not supported (data not shown), although the 918 extent of large-scale structural variation among founders in this 919 region (with the exception of CB4856, which does not show 920 unusual levels of SNP or copy number variation) is unknown. 921 Setting aside potential technical artifacts, the locus may rep- 922 resent a hitherto undetected recombination hotspot (whether 923 through attraction, or suppression of observed recombination 924 elsewhere on the chromosome), a site of rampant gene conver- 925 sion, or the focus of early and sustained selection during the 926 initial intercross phase (potentially epistatic in nature, see Neher ${ }_{927}$ and Shraiman (2009)). Earlier work proposed that evolution of 928 this region may have involved a recombination rate modifier 929 (through gene conversion) during the first 140 generations of 930 experimental evolution in order to explain the observed excess 931 haplotype diversity (see discussion and Figures $\mathrm{S} 4$ and $\mathrm{S} 5$ of $\mathrm{Ch}-{ }_{932}$ elo and Teotónio (2013)). In contrast, chromosome V, which has 933 been the focus of a recent large-scale selective sweep (Andersen 934 et al. 2012), shows more variable expansion across sub-panels 935 suggestive of ongoing selection (Figure S4).

\section{Population stratification}

We examined additional genetic structure in the CeMEE RIL panel stemming from the inclusion of distinct sub-panels of RILs that vary in experimental evolution histories. In the context of QTL mapping, this genetic structure represents nuisance variation that can bias estimates of heritability if unknown factors covary with the trait of interest, structure that is causally associated with a trait, or non-causal structure due solely to population stratification.

To gauge the extent of population stratification we compared the results of supervised and unsupervised discriminant analysis of principal components (DAPC; Jombart (2008)), which partitions within and between group variation, using either known or inferred populations, based on linear combinations of principal components. By selection of discriminant functions that best predict known CeMEE sub-panel membership, it is clear that the varied evolutionary history has, unsurprisingly, generated significant genetic structure. The number of principal components selected by cross-validation that best predicts population membership is 40 , which together explain $25 \%$ of the variance (though only a fraction of these components are significantly associated, considered singly or in pairs, see 3). Unsupervised DAPC, which infers groups based on variance minimization and model penalization criteria ( $k$-means clustering, BIC), selected 58 clusters which best explain the data ( $\triangle$ BIC $<1$ over this range). These corresponded significantly with sub-panel identity (e.g., $p$ $=0.036$ at $k=5$, permutation test), although the rate of successful assignment was low $(36 \%$ at $k=5)$. This suggests that genetic structure within, as well as between sub-panels, is significant.

\section{Heritability and predictability of fitness-proximal traits}

We measured two traits that are important components of fitness 969 - the fertility and size of young adult hermaphrodites - and thus 970 represent challenging case studies for mapping of complex traits 971 in the panel (Poullet et al. 2016). The traits are correlated (Fig- 972 ure S1), and vary extensively in the CeMEE RILs: hermaphrodite 973 fertility varies more than five-fold, size varies more than three- 974 fold (Figure 7).

Under the uncommonly met assumptions of complete tag- 976 ging of causal variation and uniform linkage, narrow sense her- 977 itability $\left(h^{2}\right)$ can be estimated from phenotypic and additive 978 genetic covariances (Henderson 1975; Robinson 1991; Yang et al. 979
2010; Speed et al. 2012; de los Campos et al. 2015). Estimates, assuming appreciable heritability, are influenced by the extent to which markers reflect the genetic architecture of the trait in the population under study, and the method by which similarity is defined from them (reviewed in Speed and Balding (2015), and see Materials and Methods). Different covariance metrics can therefore provide useful information on the genetic basis of complex traits, such as partitioning chromosomal contributions, the frequency distribution of causal variants, and the proportion of epistatic variance, without the statistical limitations (and precision) of GWAS that attempt to explain phenotypic variance as the sum of individually significant additive marker effects. As emphasized by Speed and Balding, genomic heritability estimation is best viewed as a model-fitting exercise, the problem being to find the most appropriate measure of genetic similarity for the trait, population and genetic data in question, and the answer being the most likely estimate of the contribution of genetic variance to trait variance given the data.

Repeatability, genomic heritability and prediction While RIL repeatability (an upper bound on broad sense heritability, $H^{2}$, under certain assumptions (Dohm 2002)) for both traits was relatively high -0.76 for fertility and 0.80 for size - genomic heritability estimates for trait coefficients with a simple additive genetic similarity matrix based on the probability of allele sharing at all markers, equally weighted, were not significantly different to 0 (likelihood ratio test; not shown). This suggested that genome-wide genotypic similarity is poorly correlated with causal variation for these traits, potentially due to variable LD or epistatic cancellation. We thus examined alternative measures of genetic similarity to address the apparent lack of additive genomic heritability, comparing model predictive power $\left(r^{2}\right)$ by leave-one-out cross-validation (see Materials and Methods).

Heritability estimates and prediction accuracy are summarized in Table 1, comparing the simplest models - additive (A) only, or additive + additive-by-additive $\left(\mathrm{A}^{2}\right)$ genetic covariance at the genome level - and the most predictive models for each trait. Given relatively high variance in relatedness, we are powered to detect large differences in additive heritabilities despite modest sample sizes for analysis of this kind, although the differences between individual models are generally minor. For fertility, with just 227 lines we have $50 \%$ power to reject $h^{2}=0$ if $h^{2}=0.38$, and $>95 \%$ power at our estimate of $H^{2}$ (at a significance level of 0.05), while for size, the corresponding values are $50 \%$ power at $h^{2}=0.35$ and $>99 \%$ power at repeatability (based on the best performing measure of additive similarity for each trait; Visscher et al. (2014)). Given the multiplicative scaling of epistatic similarity, low power is unavoidable.

While phenotype prediction accuracy is generally poor, some broad trends are apparent in the ranking. Additive heritability based on LD-weighted markers was relatively high for size (0.58), though less so for fertility (0.24). In neither case was additive similarity alone the best predictor of phenotype, however. Nine of the top 10 models for fertility all incorporated epistasis in some form, with the best of these giving $57 \%$ improvement over the best additive model. For size, the advantage was less clear: three of the top four models included epistasis, though the performance differential between the best epistatic and additive models was only $3 \%$.

Notably, partitioning of the genome based on recombination rate domains performed well for both traits, and was the preferred model for fertility. In general, model type was more influential on prediction than allele frequency scaling $(\alpha)$, how- 
bioRxiv preprint doi: https://doi.org/10.1101/120865; this version posted March 27, 2017. The copyright holder for this preprint (which was not certified by peer review) is the author/funder, who has granted bioRxiv a license to display the preprint in perpetuity. It is made available under Heritability and predictability of fitness-proximal traits aCC-BY 4.0 International license.
traits
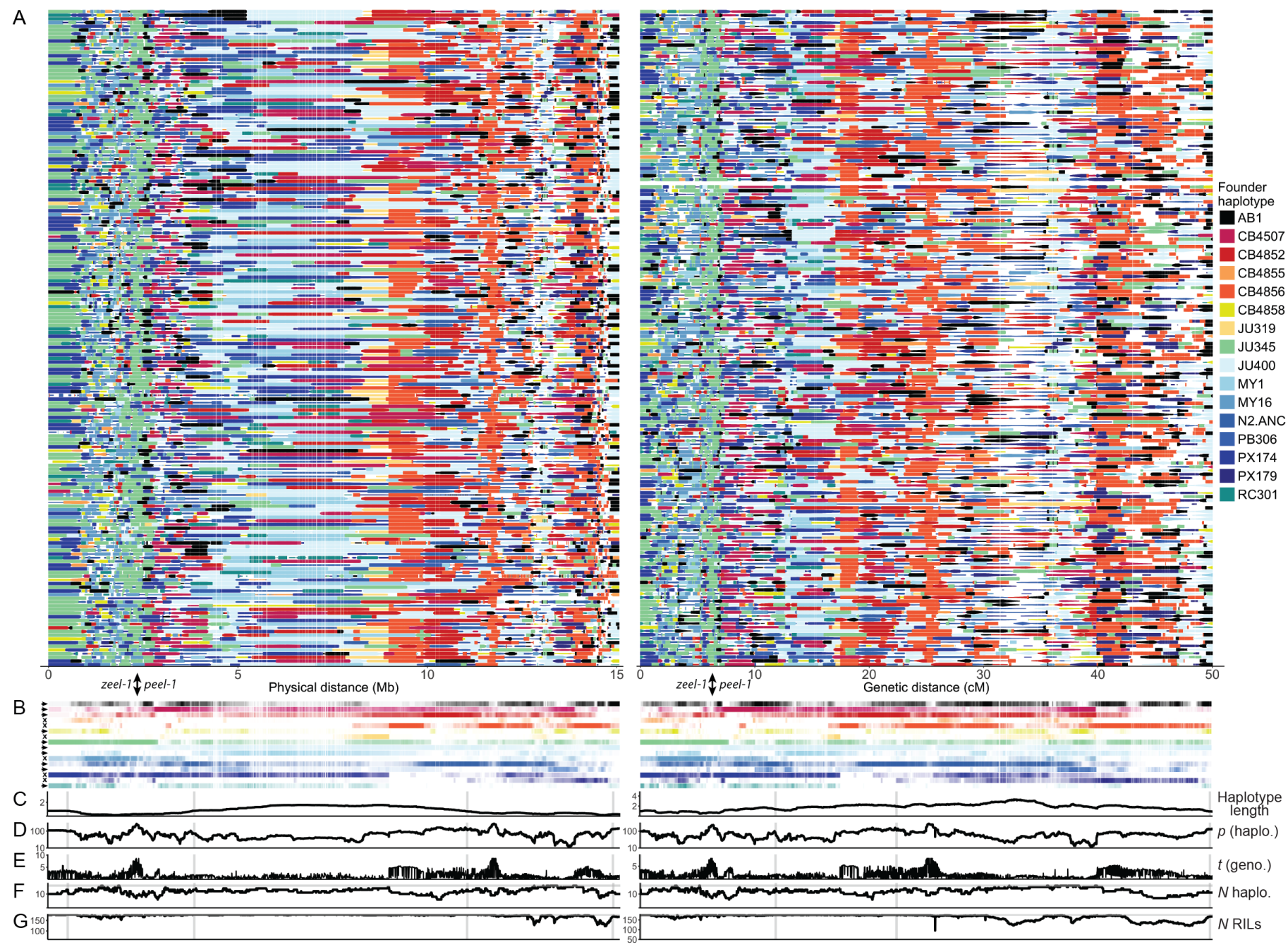

(a) Chromosome I

Figure 5 A140 RIL founder haplotype reconstruction and structure for chromosomes I, IV and X. A. Founder haplotypes reconstructed for the A140 RILs shown in physical and genetic distances. Each plotted point is a marker, with its size scaled by posterior probability (minimum 0.2). Founder contributions are summarized below in B. Loci discussed in the text are indicated: the zeel1/peel-1 incompatibility on the left arm of chromosome I (haplotype compatibility group, either experimentally tested in Seidel et al. (2008) or determined here from genotype data, is indicated below as an arrowhead for Bristol (N2) or an x for Hawaii (CB4856); extreme haplotype differentiation within a piRNA cluster on the right arm and tip of chromosome IV; and the fixation of N2/CB4507 haplotypes over a large region of the $\mathrm{X}$ chromosome left arm spanning $n p r-1$, which has known pleiotropic effects on behavior and laboratory adaptation (de Bono and Bargmann 1998; Gloria-Soria and Azevedo 2008; McGrath et al. 2009; Andersen et al. 2014). C-G show summary statistics evaluated at $5 \mathrm{~Kb}$ or $0.01 \mathrm{cM}$ resolution, with vertical scales for each metric fixed across chromosomes, and the positions of recombination rate boundaries inferred for the N2×CB4856 RIAILs (Rockman and Kruglyak 2009) indicated with shaded bars. C. Haplotype length; mean length extending from the focal position. D. $p$ (haplo.); test of reconstructed founder haplotype proportions, relative to expectation based on reconstruction frequency from $\mathrm{G}_{150}$ simulations $\left(-\log _{10}(p)\right.$ from a $\chi^{2}$ goodness-of-fit test). E. $t$ (geno.); change in allele frequency from the founders (absolute value of Welch's $t$ statistic for founder vs. RIL genotype counts). F. $N$ haplo.; the number of unique founder haplotypes detected at each position, with the maximum value of 16 indicated. G. N RILs; the number of RIL haplotypes reconstructed at each interval ( $>0.2$ posterior probability), with the maximum value of 178 indicated.

ever within models, negative values of $\alpha$ (rarer alleles having 987 larger effects) were generally preferred for size, and positive for 988 fertility, suggesting the frequency spectrum of causal variants 989 for the two traits varies in the RILs.

\section{Effects of population stratification on heritability estimation}

Given the stratified nature of the CeMEE panel, we tested for ef- 992 fects on heritability estimation in three ways. First, we estimated 993 heritability for individual sub-panels (best additive models only). Although highly uncertain given the very small sample sizes, estimates were positive for two of the three sub-panels for adult body size and for both of two sub-panels tested $(n>50)$ for fertility, spanning the reported values for all lines.

Second, we estimated within sub-panel heritability by fitting within population means as covariates (best $\mathbf{A}$ and $\mathbf{A}+\mathbf{A}^{2}$ mod- 
bioRxiv preprint doi: https://doi.org/10.1101/120865; this version posted March 27, 2017. The copyright holder for this preprint (which was not certified by peer review) is the author/funder, who has granted bioRxiv a license to display the preprint in perpetuity. It is made available under
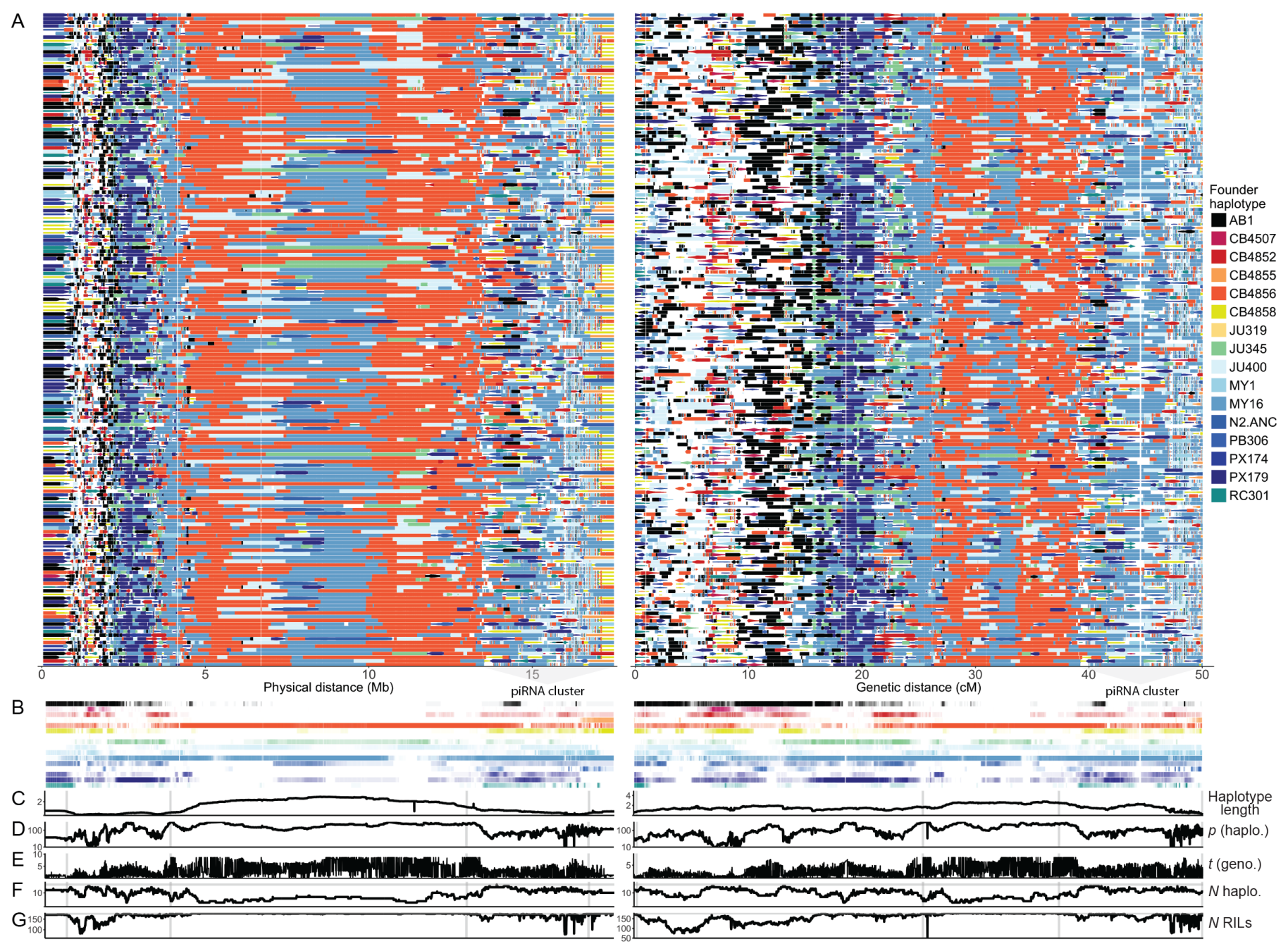

(b) Chromosome IV

els). For adult body size, where GA RILs are significantly larger 1018 than other panels, this reduced estimated heritability to 0.15 (A) 1019 and $0.38\left(\mathbf{A}+\mathbf{A}^{2}\right.$, with $\left.\mathbf{A}^{2}=0.30\right)$. Fertility, for which trait values 1020 vary only weakly with sub-panel, was largely unchanged at 0.451021 (A) and (the unreasonably high, and uncertain) estimate of 1.441022 (SD 0.75) for $\mathbf{A}+\mathbf{A}^{2}$, with a dominant contribution from epistasis. 1023

Third, we applied the method of Yang et al. (2011), developed ${ }^{1024}$ for unrelated human populations, which compares the sum ${ }_{1026}$ of heritabilities estimated for single chromosomes to that of a ${ }_{1027}$ model fitting all chromosomes jointly. In the former case, genetic ${ }_{1028}$ correlations across chromosomes due to population structure ${ }_{1029}$ will result in $\sum h_{C(\text { single })}^{2}>h_{C}^{2}$, since the genotype of one chro- ${ }_{1030}$ mosome will be predictive of that of others, while fitting all chromosomes jointly gives independent conditional estimates. The reasonable underlying assumptions are that structure is ${ }^{1031}$ more significant between than within populations, and is not 1032 causally associated with phenotypic variance, although the latter ${ }_{1033}$ might not hold for fitness-proximal traits. Comparing the sum 1034 of heritability estimates from samples of half the chromosomes 1035 $\left(\sum h^{2} / 2\right)$ to that from all chromosomes (additive similarity only), 1036 results suggested stratification may contribute significantly to 1037 our estimates for size, with mean $\sum h^{2} / 2=0.72$ (contributing $20 \% 1038$ of the total given $h^{2}=0.60$ for a joint chromosome model), but not 1039 for fertility (mean $\sum h_{/ 2}^{2}<h^{2}$ ). Fitting up to 80 principal compo- 1040 nents as covariates for size failed to bring this ratio to equality, but progressively eroded the heritability estimate (minimum $10 \%$ inflation for $80 \mathrm{PCs}, h^{2}=0.30$ ), while fitting three DAPCs (based on the top 40 PCs) fully accounted for the difference (mean $\sum h^{2}{ }^{2}=h^{2}=0.39$ ). Notably, performing the same analysis within sub-panels, however, gave a similar level of 'inflation" for size within the largest group of RILs $(28 \%)$, suggesting that structure not associated with sub-panel is also influential.

The above analyses lead us to conclude that results presented in Table 1 for fertility are robust, while those for adult size are somewhat less so. The extent of inflation, however, is unlikely to be as severe as indicated by disjoint genome partitioning, and no covariates were fit for subsequent analyses.

\section{GWAS}

QTL mapping power and precision We first explored the characteristics of the CeMEE panel relevant to mapping quantitative traits. We carried out association tests by linear mixed effects model on simulated phenotypes, varying the effect size of causal variation and the degree of polygenicity (see Materials and Methods). The panel reaches $50 \%$ power for an allele explaining 0.047 of the phenotypic variance (permutation $5 \%$ significance threshold of $p<1.62 \times 10^{-6}$ ), with recall (\% true positives) greater than $50 \%$, (Figure 6). When detected, the median QTL support inter- 
bioRxiv preprint doi: https://doi.org/10.1101/120865; this version posted March 27, 2017. The copyright holder for this preprint (which was not certified by peer review) is the author/funder, who has granted bioRxiv a license to display the preprint in perpetuity. It is made available under GWAS aCC-BY 4.0 International license.
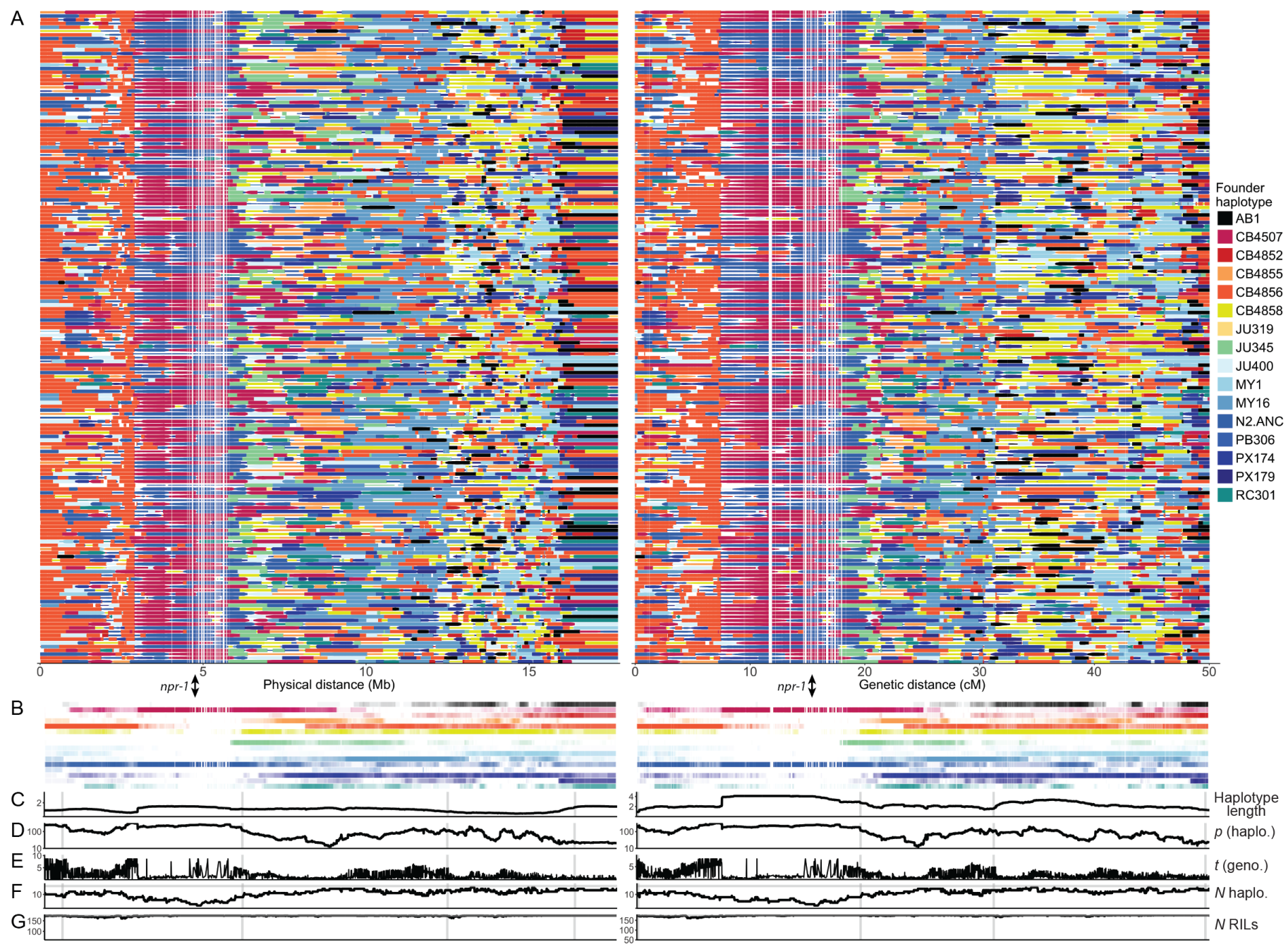

(c) Chromosome X

val (a drop in LOD of 2) spans $<10 \mathrm{~Kb}$ for variants explaining 1005 $>2.5 \%$ of trait variance. Given an average gene size of approxi- 1066 mately $5 \mathrm{~Kb}$ in C. elegans $\mathrm{N} 2$, including intergenic sequence, the 1067 CeMEE reaches sub-genic resolution for alleles of moderate ef- 1068 fect $(>10 \%$ ), yielding high mapping precision (Figure 6). We note 1069 that our simulations are unbiased with respect to chromosomal 1070 location, while causal variation for many traits may be enriched 1071 on the highly recombinant arms, so these estimates are likely to be conservative.

1D mapping of fertility and size We carried out single marker ${ }_{1074}$ genome-wide association tests by linear mixed effects model, 1075 controlling for genome-wide relatedness using the most predic- 1076 tive LD-weighted additive genetic similarity matrix for each 1077 trait (see above). Based on permutation thresholds, no single 1078 marker reached significance in either case $(\alpha=0.1$ thresholds 1079 $=4.38 \times 10^{-6}$ and $5.57 \times 10^{-7}$ for size and fertility, with mini- 1080 mum observed $p$-values of $2.8 \times 10^{-5}$ and $7.23 \times 10^{-5}$ respec- 1081 tively; Figure 7). For size, $p$-values were moderately inflated 1082 at the high end, with a number of regions approaching signif- 1083 icance, but were strongly deflated for fertility, consistent with 1084 model misspecification. Results were largely independent of 1085 the method used to define similarity or, for fertility, whether 1086 correction for relatedness was applied at all (Figure S5). LD ${ }_{1087}$ score regression, a related approach that explicitly assumes an 1088 infinitesimal architecture (Bulik-Sullivan et al. 2015), gave further support for extensive polygenicity with effects distributed across the genome (again, mostly clearly for fertility; Figure S6). Given significant heritabilities for both traits, and the results of GWAS simulations, the absence of individually significant associations suggests architectures comprising many variants with additive effects explaining $<5 \%$ of the phenotypic variance.

2D mapping of additive-by-additive interactions Given suggestive evidence for epistasis from variance decomposition and a lack of individually significant additive effects by 1D mapping, we sought to identify interactions by explicitly testing pairs of markers. As summary statistics we retained the ANOVA interaction $F$ statistic, as well as the sum of values for each marker across all tests for a chromosome pair (thresholded at $F>0,8$ and 16 , the latter corresponding approximately to the most significant $1 \mathrm{D}$ associations seen). At a significance level of $\alpha=0.1$ we detect four interactions (between seven loci) for fertility and two for size, with modest marginal additive effects (Figure 8 ; best singlelocus statistics per pair ranging $p=9.1 \times 10^{-3}-9.9 \times 10^{-5}$ for fertility and $p=1.1 \times 10^{-3}-9.9 \times 10^{-6}$ for size). The variance explained by each pair, considered individually, is high: $12-15 \%$ for fertility and $7-8 \%$ for size, and a joint linear model explains $32 \%$ and $15 \%$ of the phenotypic variances.

By summing interaction scores in 1-dimensional space to test 
bioRxiv preprint doi: https://doi.org/10.1101/120865; this version posted March 27, 2017. The copyright holder for this preprint (which was not certified by peer review) is the author/funder, who has granted bioRxiv a license to display the preprint in perpetuity. It is made available under

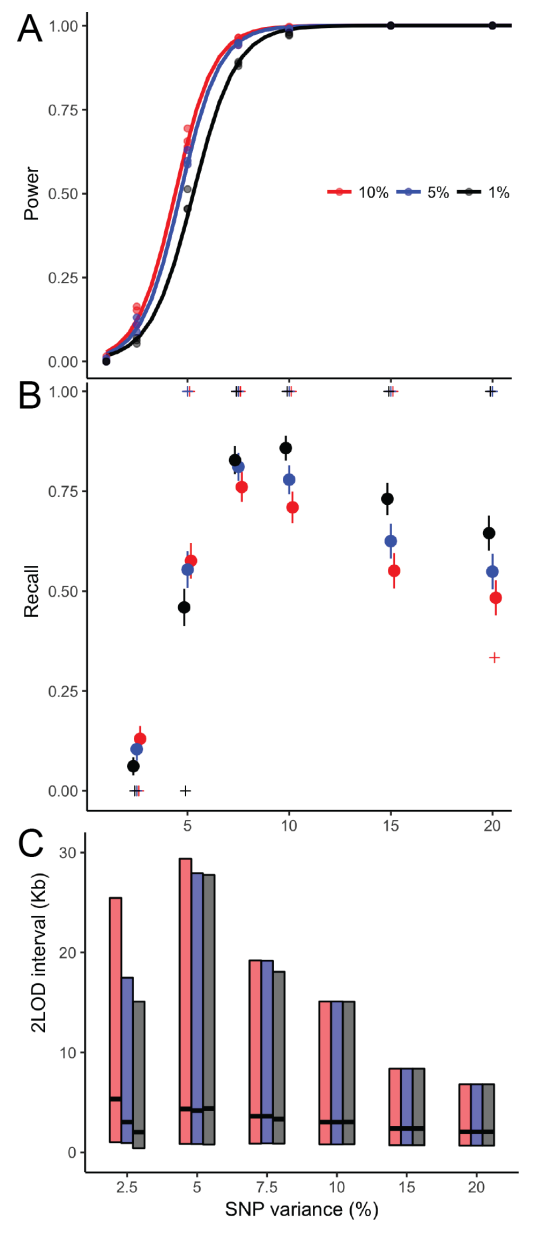

Figure 6 Additive QTL mapping simulations. Detection power 1097 (A), recall (B) and resolution (C; 2-LOD drop interval size for 1098 detected QTL) from single QTL simulations for the full map- 1099 ping panel of 507 lines, as a function of detection threshold $\quad 1100$ (significance at 0.01, 0.05 and 0.1 ) and phenotypic variance ex- 1101 plained by the simulated QTL. Total heritability of simulated 1102 phenotypes is twice that of the focal QTL, with the polygenic 1103 contribution spread over 10, 100 or 1000 background mark- $\quad 1104$ ers (plotted in A, combined in B and C). In B, points are mean 1105 \pm standard error. Recall declines with SNP variance at high levels as chance associations reach significance, although the 1106 median value (+ symbols) is 1.0 at $5 \%$ significance for variants ${ }^{1107}$ that explain $\geqslant 7.5 \%$ of trait variance.

\begin{tabular}{cccccc}
\hline Trait & GSM & $\alpha$ & $r^{2}$ & $\hat{h}^{2}(\mathrm{SD})$ & $L R$ \\
\hline Size & $\mathrm{A}$ & -0.5 & 0.073 & $\mathrm{~A} 0.58(0.14)$ & 10.8 \\
& $\mathrm{~A}+\mathrm{A}^{2}$ & $\mathbf{- 0 . 5}$ & $\mathbf{0 . 0 9 3}$ & $\mathbf{A ~ 0 . 5 7 ( 0 . 1 5 )}$ & $\mathbf{1 0 . 9}$ \\
& & & & $\mathrm{A}^{2} \mathbf{0 . 2 1}(\mathbf{0 . 5 1 )}$ & \\
\hline Fertility & $\mathrm{A}$ & 1 & 0.012 & $\mathrm{~A} 0.24(0.24)$ & 0.01 \\
& $\mathrm{~A}+\mathrm{A}^{2}$ & 1 & 0.029 & $\mathrm{~A} 0.36(0.21)$ & 2.67 \\
& & & & $\mathrm{~A}^{2} 1.24(0.87)$ & \\
& $\left(\mathbf{A}+\mathbf{A}^{2}\right)_{\text {rec }}$ & $\mathbf{1}$ & $\mathbf{0 . 0 6 4}$ & $\mathbf{A}_{\text {arm }} \mathbf{0 . 4 4}(\mathbf{0 . 1 8})$ & $\mathbf{6 . 9 8}$ \\
& & & & $\mathbf{A}_{\text {cen. }} \mathbf{0 . 0 2}(\mathbf{0 . 0 7 )}$ & \\
\hline
\end{tabular}

Table 1 Genomic heritability estimates

Results are shown for additive (A) and additive-by-additive $\left(\mathrm{A}^{2}\right)$ genetic similarity matrices (GSM), and for the most predictive model tested (if neither of the above), shown in bold. $\alpha$ is the scaling parameter from (Speed et al. 2012), which determines the effect size expectation for markers as a function of allele frequency, where 0 is unweighted and smaller values assign greater weight to rare alleles. Unconstrained REML estimates and standard deviations are shown for components that were $>0$ at convergence. $L R$ is improvement over the null model (likelihood ratio). $\left.\mathrm{A}+\mathrm{A}^{2}\right)_{\text {rec }}$ is additive and additive-byadditive similarity at the level of recombination rate domains (tips, arms and central domains). s.t. dire are shared with IV:1,914,315, and among all 26 interactions involving these two sites ( $F>16$ for either), interactions statistics are uncorrelated $(r=-0.15, p=0.49)$. Nevertheless, experiment will be required to test these loci independently.

IV:1,914,315 is found within an intron of egl-18 (encoding a GATA transcription factor), while IV:1,894,021 falls within the large intergenic region between egl-18 and egl-4 (encoding a cyclic-GMP-dependent protein kinase thought to act in the TGFbeta pathway), both of which vary in coding and UTR sequence among founders, and have numerous known phenotypes from classical induced mutations and RNAi spanning the gamut of behavior, development and reproduction. Their eponymous phenotype, egg-laying abnormal (Egl), is retention of oocytes and embryos, a phenotype selected during experimental evolution 16 in which embryos were extracted each generation by bleaching 1117 (Poullet et al. 2016). 


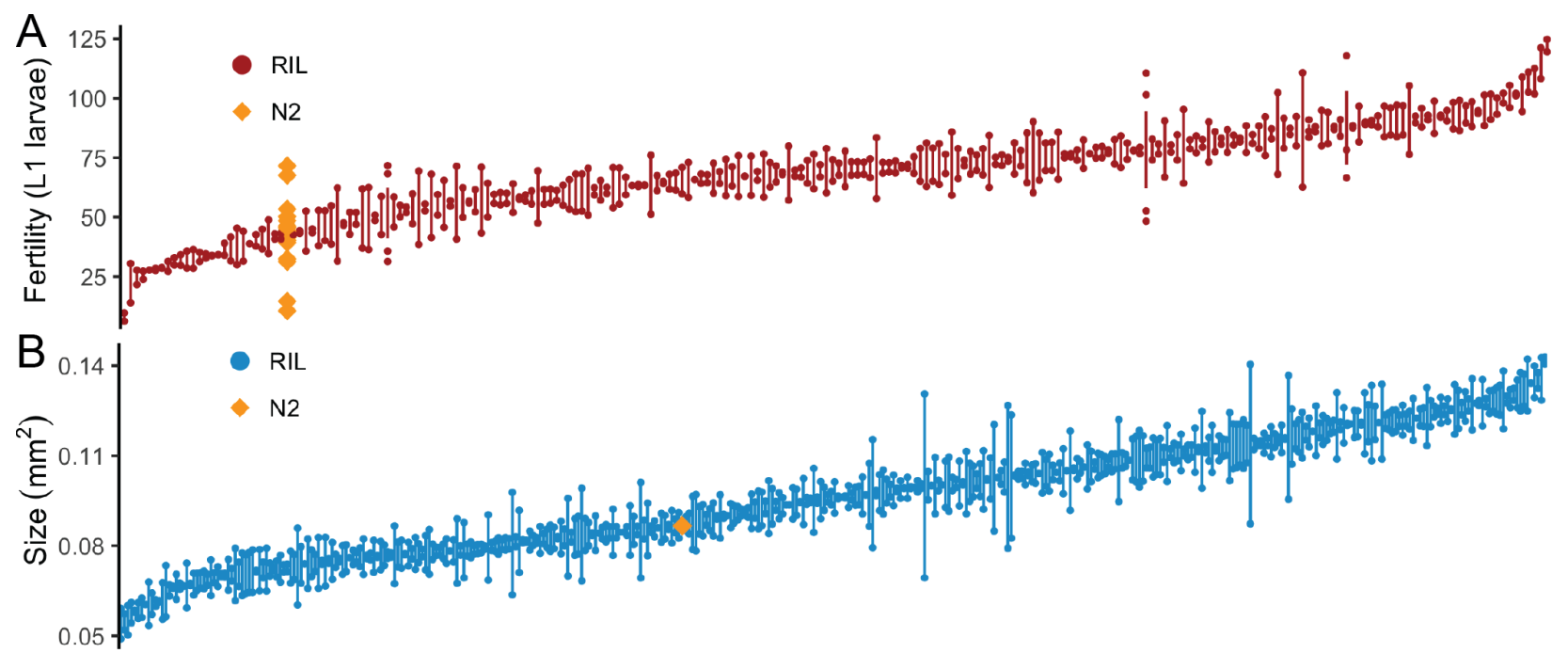

C

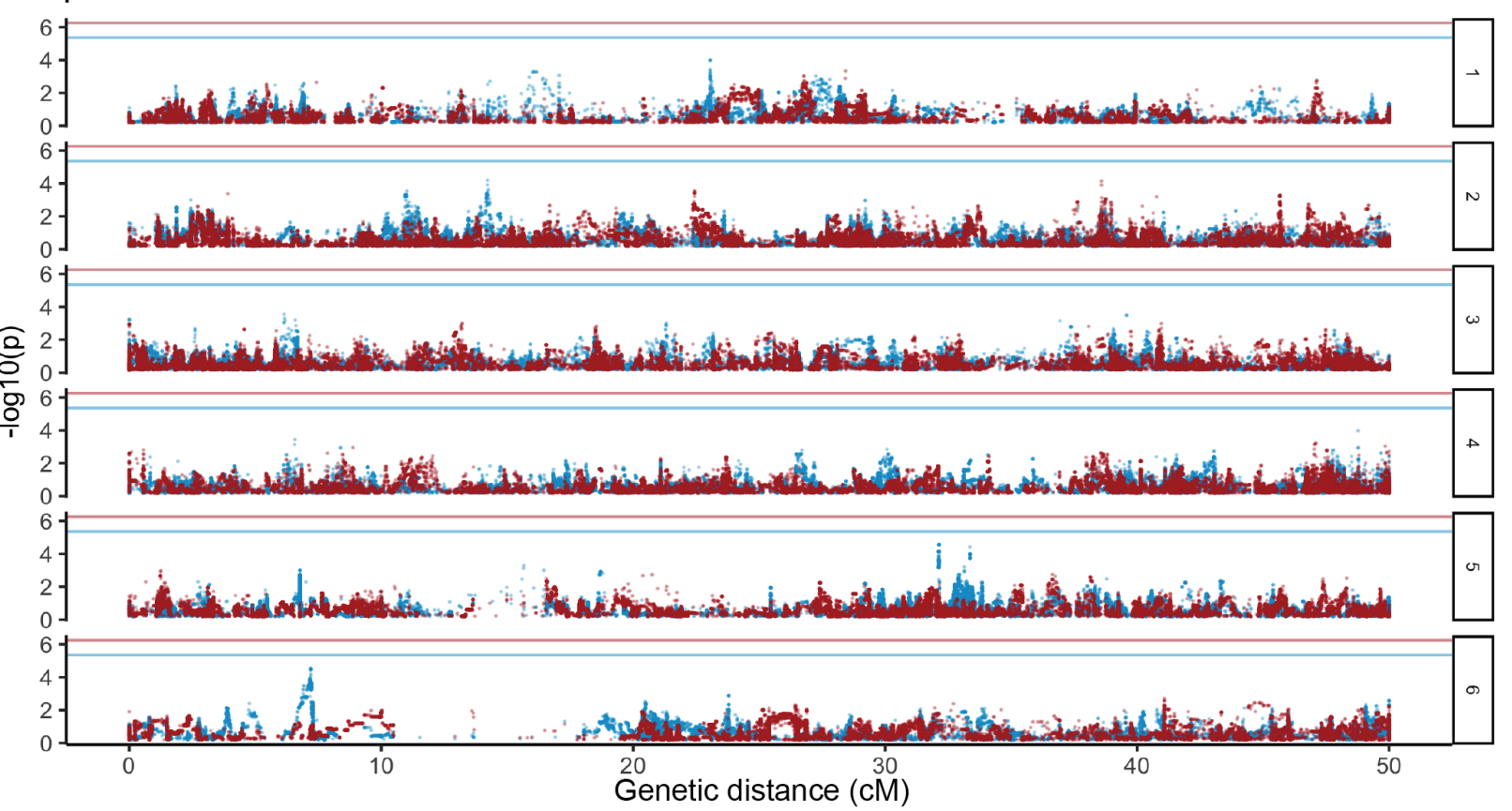

Figure 7 1D GWAS. A-B. Trait value distributions across RILs (replicate means; bars show data range or the standard error for samples with $>2$ replicates) and (C) single-SNP association results for fertility and adult body size (colors as above). Values for the reference N2 strain are shown for comparison. Note that values are raw replicate means on the original scale, and so include all sources of technical variation (unlike model coefficients used for mapping). 


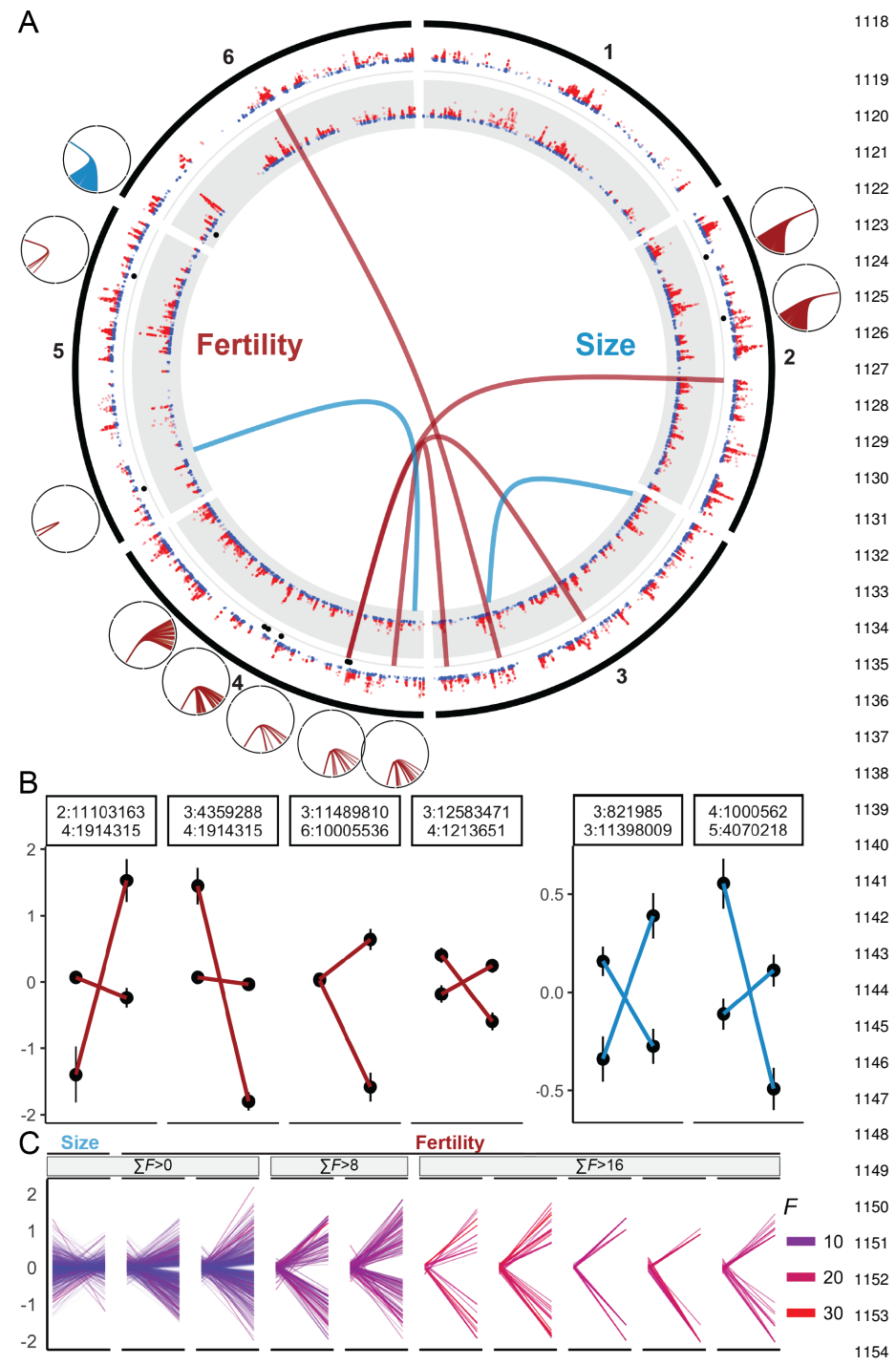

Figure 8 Strong sign epistasis and highly polygenic interactions contribute to trait variance. A. The distribution of significant interactions for fertility and size (genetic distance). Pairwise interactions are plotted over 1D GWAS test statistics $(-\log 10(p)>1)$ for each trait. Markers with a significant excess of summed interactions for a given chromosome pair are indicated with black points, and the chromosome identities and locations of interacting loci are shown as smaller plots at their approximate positions. 2D sum tests are directed interactions between a single focal marker, and all other markers on one other chromosome, with the sum of interaction scores reaching significance $(\alpha=0.1)$ under a null permutation model. Note interactions between chromosome V:3,145,783 and 16 loci on the right tip of chromosome IV are clustered over a physical interval of $0.44 \mathrm{Mb}$ (in weak LD) and appear as a single link at this resolution. B. Genotype class trait means ( $\pm \mathrm{SE}$ ) for significant pairs (fertility in red, size in blue). C.

Genotype class trait means for all individual pairs that contribute to significant summed interactions, at each of the three evaluated $F$ statistic thresholds (interactions significant at $F>0$ are filtered to $F>2$ for plotting). Line color and intensity is scaled by $F$ for each constituent interaction. Strong sign epistasis (including weak reciprocal sign epistasis) is the prevalent epistatic mode.

\section{Conclusions}

We have described the generation, characterization and application of the first multiparental mapping panel for the model organism C. elegans. Drawing on effectively 260 generations of moderate population sizes and predominant outcrossing during laboratory culture, full reference-based genome sequencing of the 16 inbred wild founders, and dense genotyping of the RILs, the CeMEE panel yields gene level mapping resolution for alleles of $5 \%$ effect or greater. For traits such as gene expression, for which the proportion of variance explained by local variation is typically upwards of $20 \%$ (e.g., Brem and Kruglyak (2005); Rockman et al. (2010); King et al. (2014), the majority of QTL intervals will dissect single genes.

While reference-based genotyping will remain a necessity for some time yet, it leaves the contribution of certain classes of genetic variation uncertain, and can hamper variant calling due to mapping bias and erroneous alignments at copy number variants. The genome of only one wild-isolate, the Hawaiian CB4856, has been assembled de novo to a high standard, revealing extensive divergence (Thompson et al. 2015). The ultimate goal of full genomes for all founders will yield both better accuracy in calculating genetic similarity, and ability to measure the phenotypic effects of this recalcitrant variation. Similarly undetermined, given RIL genotyping by mostly low coverage sequencing, is the extent and fate of novel mutations during experimental evolution. With a mutation rate of around 1 /genome/generation for SNPs, and more for multinucleotide mutations and copy number variation (Denver et al. 2004a,b; Seyfert et al. 2008; Denver et al. 2010; Phillips et al. 2009; Lipinski et al. 2011; Meier et al. 2014), the contribution of new mutations to trait variation in the RILs may well be non-negligible. Theory suggests that fixation of adaptive mutations should not be significant during experimental evolution (Hill 1982; Caballero and Santiago 1995; Matuszewski et al. 2015), but empirical evidence is mixed (Estes 2004; Estes et al. 2011; Denver et al. 2010; Chelo et al. 2013). Both of these factors would erode phenotype prediction accuracy, which, theoretically, should converge on $H^{2}$ given perfect genotyping of all causal variation and appropriate description of genetic covariance (de los Campos et al. 2015).

The native androdioecious mating system of C. elegans and the ability to archive strains indefinitely confer significant advantages to further use, bestowing almost microbial powers on a metazoan model. For one, the preservation of intermediate outbred populations means that the CeMEE is readily extensible, limited only by effective population sizes. However, RIL panels have several potential shortcomings. First, despite inbreeding during RIL construction, a nagging concern in use of RIL panels is residual heterozygosity (Barrière et al. 2009; Chelo et al. 2014), and the possibility of further evolution of genotypes and phenotypes subsequent to characterization. While heterozygosity appears to be at a low level in the CeMEE RILs, on average, it is not absent (see Materials and Methods). Importantly, however, given that lines are in stasis the opportunity for segregation during further use is both limited and known. A second concern is the possibility of inbreeding depression, particularly for fitnessproximal traits. This is a concern for predominantly outcrossing organisms (Barrière et al. 2009; Philip et al. 2011; King et al. 2012; Chelo et al. 2014), but it is also applicable to multiparental experimental evolution of C. elegans. As mentioned in the introduction, at least during the initial stage of laboratory adaptation, excess heterozygosity may have been maintained by epistatic overdominant selection, and closely linked recessive deleterious alleles 
in repulsion could be maintained by balancing selection during ${ }_{1242}$ inbreeding (Chelo et al. 2013, 2014). Assaying the $F_{1}$ progeny of ${ }_{1243}$ nested crosses among RILs may be a useful approach to estimate 1244 (or avoid) the effects of inbreeding depression (Long et al. 2014). 1245

Using subsets of the CeMEE panel, we outlined the genetics ${ }^{124}$ of two traits associated with fitness. Fertility, as defined here ${ }^{1247}$ by the experimental evolution protocol employed, is correlated ${ }^{1248}$ with hermaphrodite body size at the time of reproduction (Poul- ${ }^{1249}$ let et al. 2016). For both traits, and size in particular, additive ${ }^{1250}$ genomic heritability based on LD-weighted similarity explained ${ }^{125}$ a significant fraction of $H^{2}$, although heritability estimates were ${ }^{12}$ generally higher with the inclusion of epistatic similarity. This is consistent with a polygenic architecture with additive effects ${ }^{1253}$ below the detection limit, whether solely additive, or due to ${ }_{1254}$ weak or opposing effects of multiple interactions. Variance in ${ }_{1255}$ fitness-related traits, in particular, may be maintained despite ${ }_{1256}$ consistent selection on additive variation through a number of ${ }_{1257}$ processes, including stabilizing selection under a stable environment (Whitlock et al. 1995; Wolf et al. 2000; Barton and Keightley 2002; Phillips 2008; Hemani et al. 2013). Results from variance decomposition, phenotype prediction and interaction tests are ${ }^{1259}$ all consistent with this prediction: phenotypic variance remains ${ }^{1260}$ high, and we find support for epistasis for both traits. Notably ${ }^{1261}$ for fertility, which is expected to be well aligned with fitness ${ }^{1262}$ under the experimental evolution scheme, strong interactions ${ }^{1263}$ among four pairs of alleles with weak marginal main effects ${ }^{1264}$ jointly explain almost a third of the phenotypic variance. All six interactions detected for fertility and size are instances of sign 1265 epistasis, where the directional effect of one allele is reversed in the presence of another. Five of these represent the extreme form, reciprocal sign epistasis (the reversal is, to some extent at least, symmetric; Poelwijk et al. (2011)). Sign epistasis, in particular, has important implications for a population's capacity to adapt, by creating rugged fitness landscapes and constraining exploration of them (Weinreich et al. 2005, 2013), and for the repeatability of evolution, since the outcome of selection on the marginal additive effects of interacting alleles will be determined by their relative frequencies (Wright 1932; Whitlock et al. 1995; Phillips et al. 2000). Our tests for excess interactions among individually non-significant marker pairs additionally revealed a number of cases of highly polygenic epistasis, again, mostly for fertility. While tests of this type have the unsatisfying property of leaving the identities of the interacting partners uncertain, they have the potential to combat the loss of power that comes with explicit 2-dimensional testing (Crawford et al. 2016).

Fertility and body size at reproduction show broad-sense heritabilities that are relatively high for fitness-proximal traits (Lynch and Walsh 1998). This high heritability is likely a consequence of novel genetic variation created in the multiparental cross and realignment of selection to novel laboratory environments. While all mapping panels are synthetic systems, the mixing of natural variation and experimental evolution represents a perturbation that may have some parallels, for example, with that of a simultaneous founder event and environmental change, which can reveal novel incompatibilities and promote further differentiation (Cheverud and Routman 1996; Wolf et al. 2000). ${ }^{127}$ In this context, it will be useful to determine the directional ef- 1272 fects of epistasis on the genotype-phenotype map during further ${ }^{1273}$ evolution, as a function of recombination, a task for which the 1274 CeMEE is well suited. As in other systems such as Arabidop- 1275 sis, where similar resources exist (Weigel 2012) and epistasis for 1276 fitness-related traits has been found (e.g., Malmberg et al. (2005); 1277
Simon et al. (2008)), it will also be important to begin a comprehensive comparison of QTL for fitness traits in the CeMEE and natural populations - where linked selection coupled with predominant selfing and meta-population dynamics have generated limited, structured genetic diversity (Andersen et al. 2012; Rockman et al. 2010; Cutter 2015) - and also with mutational variances obtained in mutation accumulation experiments (Baer et al. 2005; Baer 2008; Joyner-Matos et al. 2009). Such comparisons have the potential to provide significant insights into how the distributions of QTL effects and frequencies are shaped in natural populations.

\section{Acknowledgements}

We thank J. Costa, R. Costa, C. Goy, F. Melo, H. Mestre, V. Pereira, and A. Silva for support with worm handling, sample preparation, and data acquisition; E. Andersen, M.-A. Félix, P.C. Phillips, S. Proulx, D. Speed and C. Zheng for helpful discussion.

\section{Funding}

This work received financial support from the National Institutes of Health (R01GM089972 and R01GM121828) to MVR, the Human Frontiers Science Program (RGP0045/2010) to B.S, M.R. and H.T, and the European Research Council (FP7/20072013/243285) and Agence Nationale de la Recherche (ANR-14ACHN-0032-01) to H.T.

\section{Author contributions}

CeMEE panel derivation: S.C., B.A., H.T.; sequencing and genotyping: A. P.-Q., D.R., I.C. P.A., L.N., M.R.; phenotyping: I.C., B.A, A.C.; analysis: L.N., I.C., T.G., A.D, B.S.; manuscript: L.N., M.R., H.T.

\section{Supplementary figures}

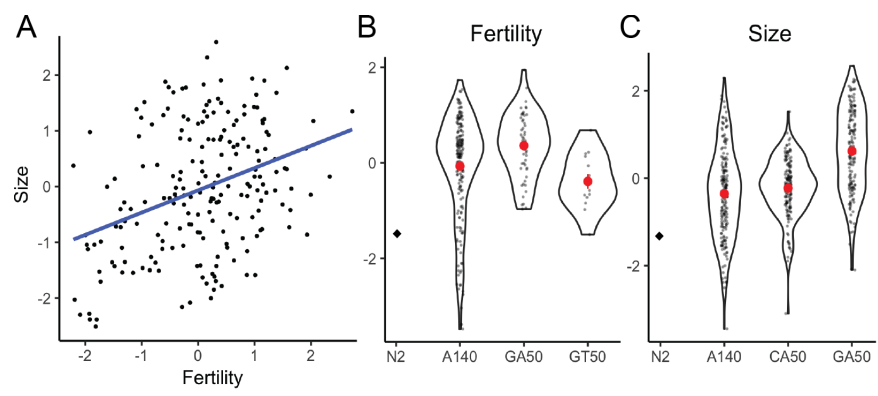

Figure S1 S1. Trait correlations and evolution. A. Fertility and size are correlated traits (Spearman's $\rho=0.318, p<5 \times 10^{-6}$ for 202 RILs with data for both traits). B-C. Trait distributions within sub-panels (density plots and mean \pm SE for centered and scaled model coefficients). The GA50 RILs are significantly larger and more fertile than A6140 RILs.

\section{Literature Cited}

Abney, M., 2015 Permutation testing in the presence of polygenic variation. Genetic epidemiology 39: 249-258.

Andersen, E. C., J. S. Bloom, J. P. Gerke, and L. Kruglyak, 2014 A variant in the neuropeptide receptor $n p r-1$ is a major determinant of Caenorhabditis elegans growth and physiology. PLOS Genetics 10: e1004156. 
bioRxiv preprint doi: https://doi.org/10.1101/120865; this version posted March 27, 2017. The copyright holder for this preprint (which was not certified by peer review) is the author/funder, who has granted bioRxiv a license to display the preprint in perpetuity. It is made available under

A
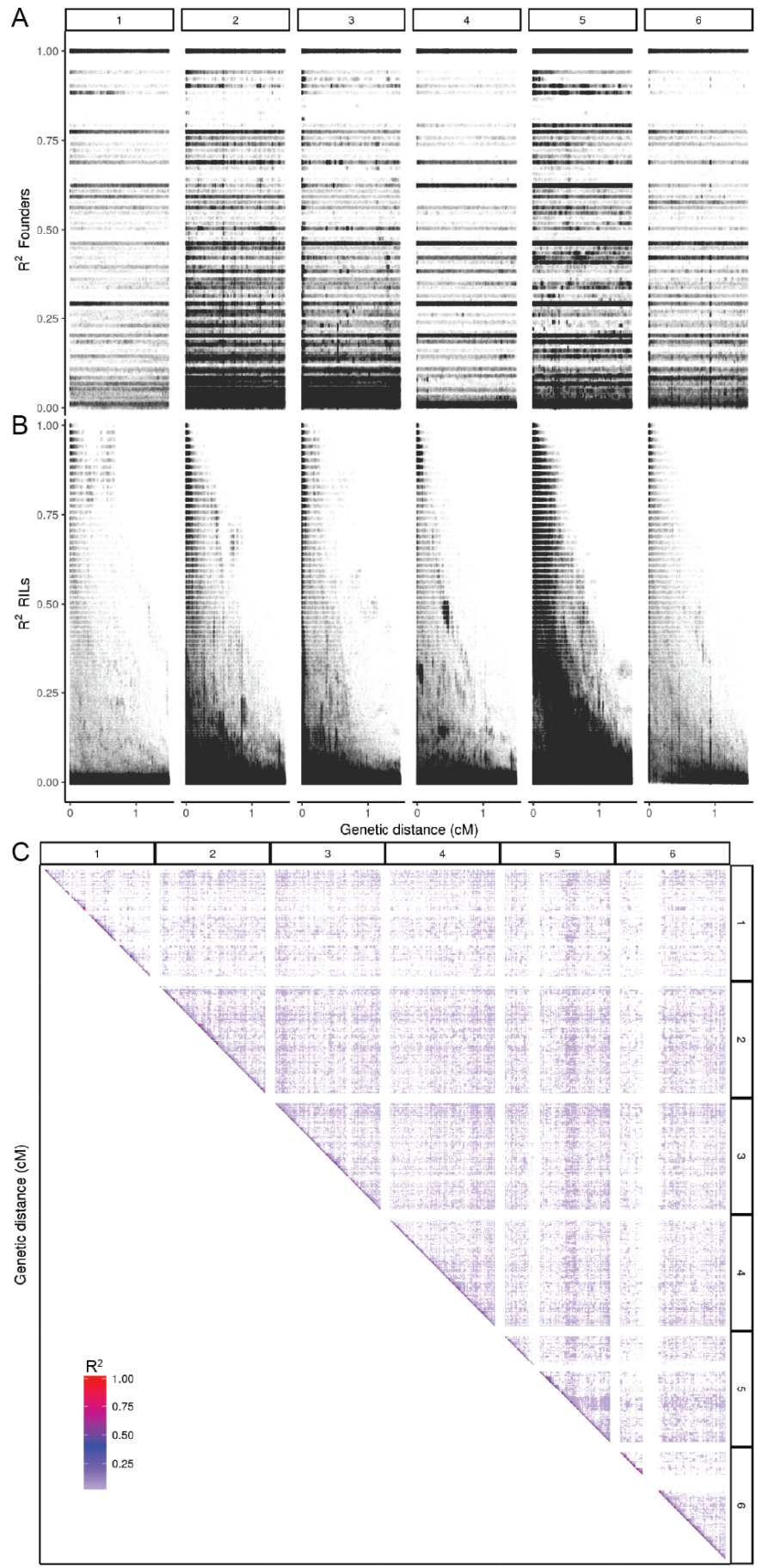

Figure S2 Local linkage disequilibrium in founders $\mathbf{A}$ and CeMEE RILs B, and across RIL genomes $\left(\mathbf{C} ; r^{2}\right.$ thresholded to $>0.01)$.

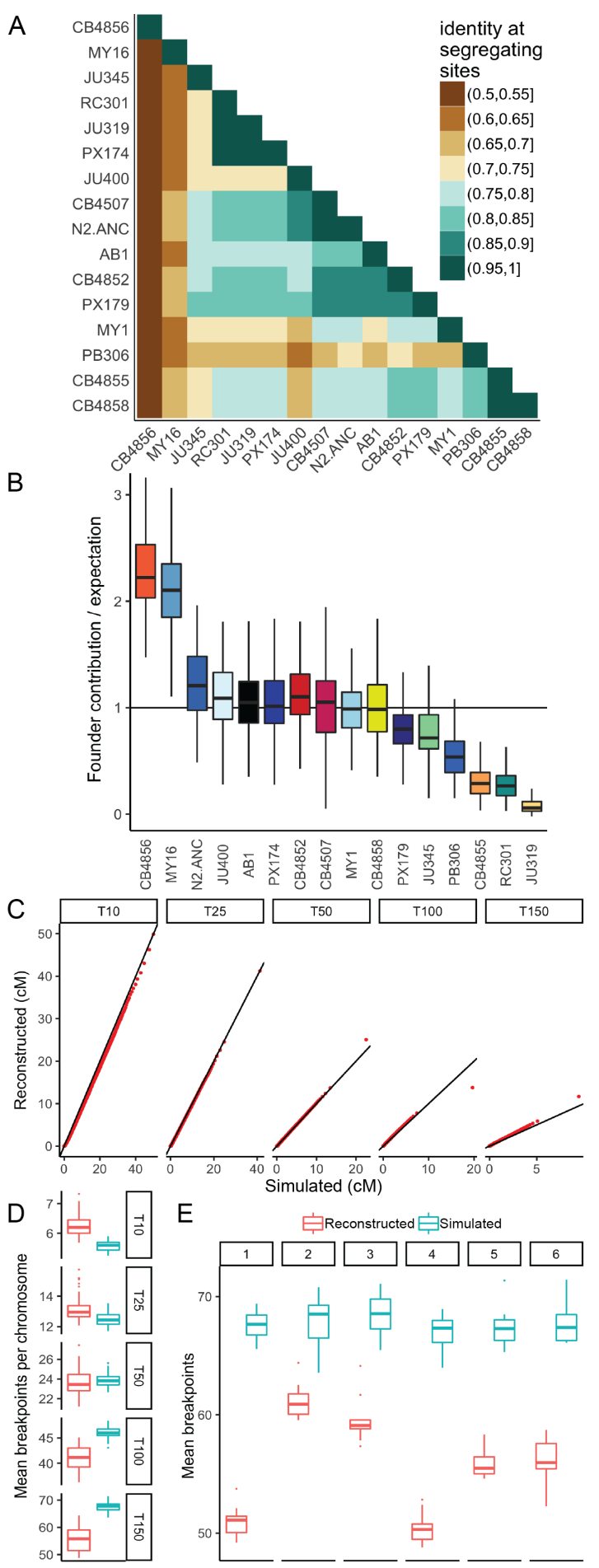

Figure S3 Summary of haplotype reconstruction. A. Genetic similarity among founders. B. Founder contributions in the CeMEE lines, relative to expectation from reconstruction of simulated recombinant genomes, accounting for bias based on haplotype uniqueness. Boxplots show median (bar), interquartile range (box) and $1.5 \times$ the data range (whiskers). C. Haplotype length quantile-quantile plots for known and reconstructed simulations. D. The number of breakpoints per chromosome per line across simulation generation. E. The number of breakpoints reconstructed by chromosome. Haplotype uniqueness varies across chromosomes: the ability to reconstruct is poorest for chromosomes I and IV. 
bioRxiv preprint doi: https://doi.org/10.1101/120865; this version posted March 27, 2017. The copyright holder for this preprint (which was not certified by peer review) is the author/funder, who has granted bioRxiv a license to display the preprint in perpetuity. It is made available under LITERATURE CITED aCC-BY 4.0 International license.

LITERATURE CITED

A

B
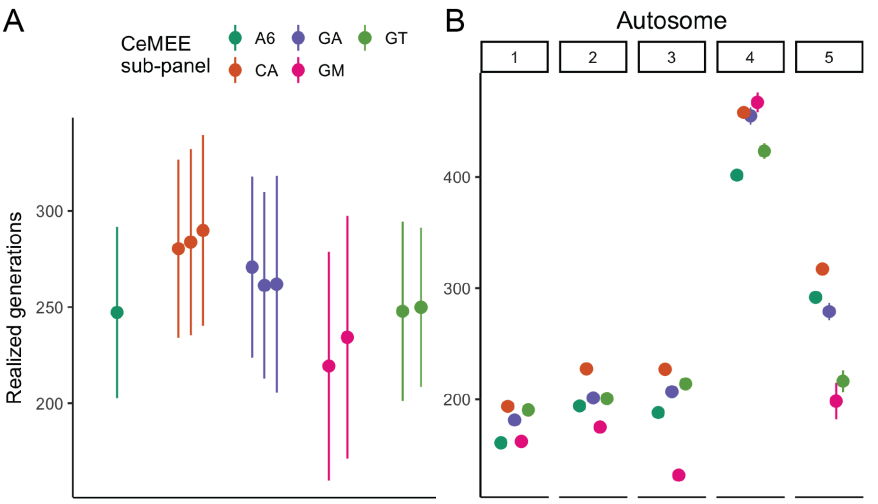

Figure S4 The number of generations of outcrossing for each $\quad{ }_{1335}$ CeMEE sub-panel (A) and chromosome (B) predicted from ${ }_{1336}$ the maximium likelihood estimate of realized map expansion ${ }_{1337}$ (Zheng et al. 2014, 2015). Andersen, E. C., J. P. Gerke, J. A. Shapiro, J. R. Crissman, 1341 R. Ghosh, J. S. Bloom, M.-A. Félix, and L. Kruglyak, $2012{ }_{1342}$ Chromosome-scale selective sweeps shape Caenorhabditis ele- ${ }_{1343}$ gans genomic diversity. Nature Genetics 44: 285-290.

Andersen, E. C., T. C. Shimko, J. R. Crissman, R. Ghosh, J. S Bloom, H. S. Seidel, J. P. Gerke, and L. Kruglyak, 2015 A ${ }_{1346}$ Powerful New Quantitative Genetics Platform, Combining ${ }_{1347}$ Caenorhabditis elegans High-Throughput Fitness Assays with a ${ }_{1348}$ Large Collection of Recombinant Strains. G3 (Bethesda, Md.) ${ }_{1349}$ 5: 911-920.

1350

Anderson, J. L., L. T. Morran, and P. C. Phillips, 2010 Outcrossing 1351 and the maintenance of males within C. elegans populations. ${ }_{1352}$ The Journal of heredity 101 Suppl 1: S62-74.

Baer, C. F., 2008 Quantifying the Decanalizing Effects of Spon- ${ }_{1354}$ taneous Mutations in Rhabditid Nematodes. The American ${ }_{1355}$ Naturalist 172: 272-281.

1356

Baer, C. F., F. Shaw, C. Steding, M. Baumgartner, A. Hawkins, 1357 A. Houppert, N. Mason, M. Reed, K. Simonelic, W. Woodard, 1358 M. Lynch, and W. W. Anderson, 2005 Comparative Evolution- ${ }_{1359}$ ary Genetics of Spontaneous Mutations Affecting Fitness in ${ }_{1360}$ Rhabditid Nematodes. Proceedings of the National Academy ${ }_{1361}$ of Sciences of the United States of America 102: 5785-5790.

Baldwin-Brown, J. G., A. D. Long, and K. R. Thornton, $2014{ }_{1363}$ The power to detect quantitative trait loci using resequenced, ${ }_{1364}$ experimentally evolved populations of diploid, sexual organ- ${ }_{1365}$ isms. Molecular biology and evolution 31: 1040-1055.

Bandillo, N., C. Raghavan, P. A. Muyco, M. A. L. Sevilla, I. T. ${ }_{1367}$ Lobina, C. J. Dilla-Ermita, C.-W. Tung, S. McCouch, M. Thom- ${ }_{1368}$ son, R. Mauleon, R. K. Singh, G. Gregorio, E. Redoña, and ${ }_{1369}$ H. Leung, 2013 Multi-parent advanced generation inter-cross ${ }_{1370}$ (MAGIC) populations in rice: progress and potential for ge- ${ }_{1371}$ netics research and breeding. Rice (New York, N.Y.) 6: 11.

Barkoulas, M., J. S. van Zon, J. Milloz, A. van Oudenaarden, 1373 and M.-A. Félix, 2013 Robustness and Epistasis in the C. ele- ${ }_{1374}$ gans Vulval Signaling Network Revealed by Pathway Dosage 1375 Modulation. Developmental Cell 24: 64-75.

Barrière, A. and M.-A. Félix, 2005 Natural variation and popula- 1377 tion genetics of Caenorhabditis elegans. pp. 1-19.

Barrière, A. and M.-A. Félix, 2007 Temporal dynamics and link- ${ }_{1379}$ age disequilibrium in natural Caenorhabditis elegans popula- ${ }_{1380}$ tions. Genetics 176: 999-1011.

Barrière, A., S.-P. Yang, E. Pekarek, C. G. Thomas, E. S. Haag, and ${ }_{1382}$ I. Ruvinsky, 2009 Detecting heterozygosity in shotgun genome assemblies: Lessons from obligately outcrossing nematodes. Genome research 19: 470-480.

Barton, N. H. and P. D. Keightley, 2002 Multifactorial genetics: Understanding quantitative genetic variation. Nature Reviews Genetics 3: 11-21.

Barton, N. H. and M. Turelli, 1991 Natural and sexual selection on many loci. Genetics 127: 229-255.

Bendesky, A., M. Tsunozaki, M. V. Rockman, L. Kruglyak, and C. I. Bargmann, 2011 Catecholamine receptor polymorphisms affect decision-making in C. elegans. Nature 472: 313-318.

Bernstein, M. R. and M. V. Rockman, 2016 Fine-Scale Crossover Rate Variation on the Caenorhabditis elegans X Chromosome. G3 (Bethesda, Md.) 6: 1767-1776.

Bloom, J. S., I. Kotenko, M. J. Sadhu, S. Treusch, F. W. Albert, and L. Kruglyak, 2015 Genetic interactions contribute less than additive effects to quantitative trait variation in yeast. Nature communications 6: 8712.

Bonhoeffer, S., C. Chappey, N. T. Parkin, J. M. Whitcomb, and C. J. Petropoulos, 2004 Evidence for positive epistasis in HIV-1. Science (New York, N.Y.) 306: 1547-1550.

Bradić, M., J. Costa, and I. M. Chelo, 2011 Genotyping with Sequenom. In Genome-Wide Association Studies and Genomic Prediction, pp. 193-210, Humana Press, Totowa, NJ.

Brem, R. B. and L. Kruglyak, 2005 The landscape of genetic complexity across 5,700 gene expression traits in yeast. Proceedings of the National Academy of Sciences 102: 1572-1577.

Buckler, E. S., J. B. Holland, P. J. Bradbury, C. B. Acharya, P. J. Brown, C. Browne, E. Ersoz, S. Flint-Garcia, A. Garcia, J. C. Glaubitz, M. M. Goodman, C. Harjes, K. Guill, D. E. Kroon, S. Larsson, N. K. Lepak, H. Li, S. E. Mitchell, G. Pressoir, J. A. Peiffer, M. O. Rosas, T. R. Rocheford, M. C. Romay, S. Romero, S. Salvo, H. Sanchez Villeda, H. S. da Silva, Q. Sun, F. Tian, N. Upadyayula, D. Ware, H. Yates, J. Yu, Z. Zhang, S. Kresovich, and M. D. McMullen, 2009 The genetic architecture of maize flowering time. Science (New York, N.Y.) 325: 714-718. Bulik-Sullivan, B. K., P.-R. Loh, H. K. Finucane, S. Ripke, J. Yang, N. Patterson, M. J. Daly, A. L. Price, and B. M. Neale, 2015 LD Score regression distinguishes confounding from polygenicity in genome-wide association studies. Nature Genetics 47: 291295.

Caballero, A. and E. Santiago, 1995 Response to selection from new mutation and effective size of partially inbred populations. I. Theoretical results. Genetical Research .

Carlborg, Ö., L. Jacobsson, P. Ahgren, P. Siegel, and L. Andersson, 2006 Epistasis and the release of genetic variation during longterm selection. Nature Genetics 38: 418-420.

Charlesworth, D. and S. I. Wright, 2001 Breeding systems and genome evolution 11: 685-690.

Chelo, I. M., S. Carvalho, M. Roque, S. R. Proulx, and H. Teotónio, 2014 The genetic basis and experimental evolution of inbreeding depression in Caenorhabditis elegans. Heredity 112: 248-254.

Chelo, I. M., J. Nédli, I. Gordo, and H. Teotónio, 2013 An experimental test on the probability of extinction of new genetic variants. Nature communications 4: 2417.

Chelo, I. M. and H. Teotónio, 2013 The opportunity for balancing selection in experimental populations of Caenorhabditis elegans. Evolution 67: 142-156.

Cheverud, J. M. and E. J. Routman, 1995 Epistasis and its contribution to genetic variance components. Genetics .

Cheverud, J. M. and E. J. Routman, 1996 Epistasis as a Source of Increased Additive Genetic Variance at Population Bottlenecks. Evolution 50: 1042. 

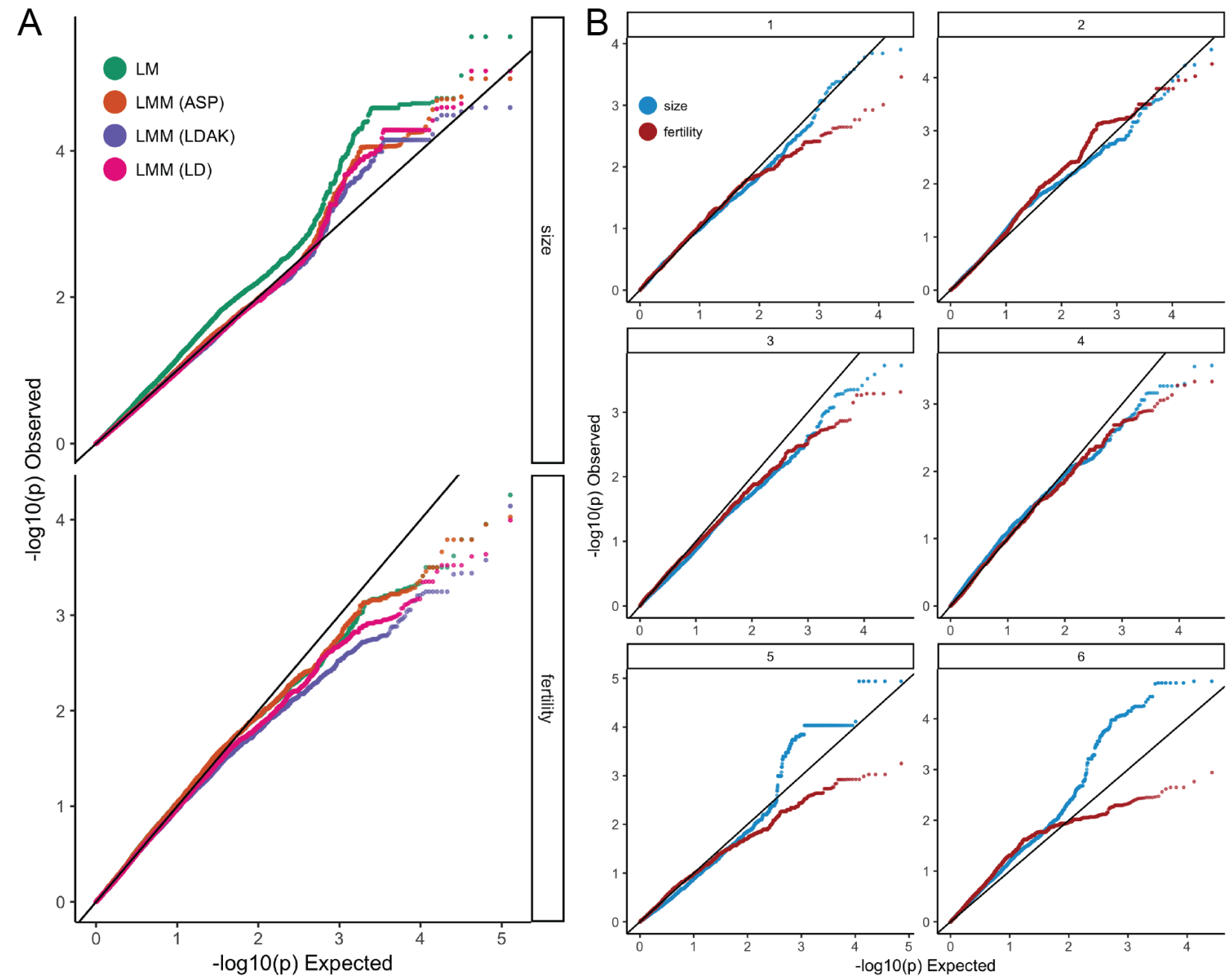

Figure S5 $p$-value quantile-quantile plots genome-wide (A), comparing the effects of relatedness corrections (where LM is linear model; LMM (ASP) is linear mixed model with relatedness based on allele sharing probability (all markers, equally weighted); LMM (LDAK) is the best performing LD-weighted similarity for each trait; LMM (LD) is based on markers pruned by local LD, but unweighted), and by chromosome (B), for the best LD-weighted similarity for each trait. While strong, spurious inflation is seen for size without polygenic correction (A), this is not seen for fertility, likely due the greater heterogeneity of trait values among sub-panels for size. Notably, deflation is seen for fertility for all models, although LD weighting introduces the strongest penalty, which may indicate a relationship between low LD and causal variation for this trait. 
bioRxiv preprint doi: https://doi.org/10.1101/120865; this version posted March 27, 2017. The copyright holder for this preprint (which was not certified by peer review) is the author/funder, who has granted bioRxiv a license to display the preprint in perpetuity. It is made available under

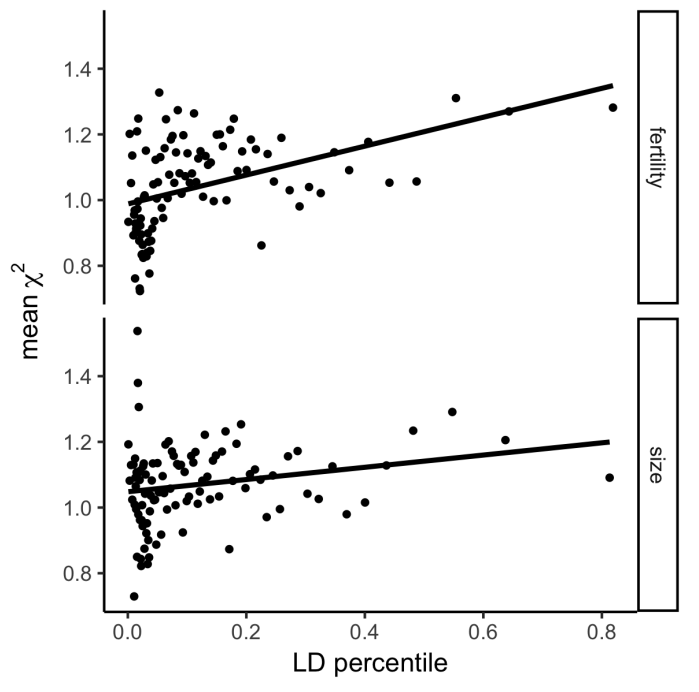

Figure S6 Fitness-proximal traits are polygenic. Regression of ${ }_{1436}$ association statistics (mean value of $\chi^{2}$ percentiles) on marker ${ }_{1437}$ LD weightings (mean of $w$ percentiles, Speed et al. (2012)) for 1438 fertility and size (after Bulik-Sullivan et al. (2015)). While there 1439 is a significant positive relationship between trait association 1440 and the amount of variation tagged by markers, fertility shows 1441 much stronger evidence of polygenicity (slope $=0.44, p=2.7 \times 1442$ $10^{-6}$, versus slope $=0.19, p=0.029$ for size).

Chirgwin, E., D. J. Marshall, C. M. Sgrò, and K. Monro, 20161446 The other 96\%: Can neglected sources of fitness variation offer ${ }_{1447}$ new insights into adaptation to global change? Evolutionary ${ }_{1448}$ Applications 10: 267-275.

Churchill, G. A., D. C. Airey, H. Allayee, J. M. Angel, A. D. Attie, 1450 J. Beatty, W. D. Beavis, J. K. Belknap, B. Bennett, W. Berret- ${ }_{1451}$ tini, A. Bleich, M. Bogue, K. W. Broman, K. J. Buck, E. Buck- ${ }_{1452}$ ler, M. Burmeister, E. J. Chesler, J. M. Cheverud, S. Clapcote, 1453 M. N. Cook, R. D. Cox, J. C. Crabbe, W. E. Crusio, A. Dar- ${ }_{1454}$ vasi, C. F. Deschepper, R. W. Doerge, C. R. Farber, J. Forejt, ${ }_{1455}$ D. Gaile, S. J. Garlow, H. Geiger, H. Gershenfeld, T. Gordon, 1456 J. Gu, W. Gu, G. de Haan, N. L. Hayes, C. Heller, H. Him- ${ }_{1457}$ melbauer, R. Hitzemann, K. Hunter, H.-C. Hsu, F. A. Iraqi, ${ }_{1458}$ B. Ivandic, H. J. Jacob, R. C. Jansen, K. J. Jepsen, D. K. John- ${ }_{1459}$ son, T. E. Johnson, G. Kempermann, C. Kendziorski, M. Kotb, ${ }_{1460}$ R. F. Kooy, B. Llamas, F. Lammert, J.-M. Lassalle, P. R. Lowen- ${ }_{1461}$ stein, L. Lu, A. Lusis, K. F. Manly, R. Marcucio, D. Matthews, 1462 J. F. Medrano, D. R. Miller, G. Mittleman, B. A. Mock, J. S. ${ }_{1463}$ Mogil, X. Montagutelli, G. Morahan, D. G. Morris, R. Mott, ${ }_{1464}$ J. H. Nadeau, H. Nagase, R. S. Nowakowski, B. F. O'Hara, ${ }_{1465}$ A. V. Osadchuk, G. P. Page, B. Paigen, K. Paigen, A. A. Palmer, 1466 H.-J. Pan, L. Peltonen-Palotie, J. Peirce, D. Pomp, M. Pravenec, ${ }_{1467}$ D. R. Prows, Z. Qi, R. H. Reeves, J. Roder, G. D. Rosen, E. E. 1468 Schadt, L. C. Schalkwyk, Z. Seltzer, K. Shimomura, S. Shou, 1469 M. J. Sillanpää, L. D. Siracusa, H.-W. Snoeck, J. L. Spearow, 1470 K. Svenson, L. M. Tarantino, D. Threadgill, L. A. Toth, W. Val- ${ }_{1471}$ dar, F. P.-M. de Villena, C. Warden, S. Whatley, R. W. Williams, 1472 T. Wiltshire, N. Yi, D. Zhang, M. Zhang, and F. Zou, 2004 The ${ }_{1473}$ Collaborative Cross, a community resource for the genetic ${ }_{1474}$ analysis of complex traits. Nature Genetics 36: 1133-1137. ${ }_{1475}$ Cook, D. E., S. Zdraljevic, J. P. Roberts, and E. C. Andersen, $2017{ }_{1476}$ CeNDR, the Caenorhabditis elegans natural diversity resource. 1477 Nucleic acids research 45: D650-D657. Cook, D. E., S. Zdraljevic, R. E. Tanny, B. Seo, D. D. Riccardi,
L. M. Noble, M. V. Rockman, M. J. Alkema, C. Braendle, J. E. Kammenga, J. Wang, L. Kruglyak, M.-A. Félix, J. Lee, and E. C. Andersen, 2016 The Genetic Basis of Natural Variation in Caenorhabditis elegans Telomere Length. Genetics 204: 371-383.

Corsi, A. K., B. Wightman, and M. Chalfie, 2015 A Transparent Window into Biology: A Primer on Caenorhabditis elegans. Genetics 200: 387-407.

Crawford, L., S. Mukherjee, and X. Zhou, 2016 Detecting Epistasis in Genome-wide Association Studies with the Marginal EPIstasis Test. bioRxiv p. 066985.

Cutter, A. D., 2004 Sperm-limited fecundity in nematodes: how many sperm are enough? Evolution 58: 651-655.

Cutter, A. D., 2006 Nucleotide polymorphism and linkage disequilibrium in wild populations of the partial selfer Caenorhabditis elegans. Genetics 172: 171-184.

Cutter, A. D., 2015 Caenorhabditis evolution in the wild. BioEssays : news and reviews in molecular, cellular and developmental biology 37: 983-995.

Cutter, A. D., A. Dey, and R. L. Murray, 2009 Evolution of the Caenorhabditis elegans genome.

de Bono, M. and C. I. Bargmann, 1998 Natural variation in a neuropeptide $\mathrm{Y}$ receptor homolog modifies social behavior and food response in C. elegans. Cell 94: 679-689.

de los Campos, G., D. Sorensen, and D. Gianola, 2015 Genomic Heritability: What Is It? PLOS Genetics 11: e1005048.

de Visser, J. A. G. M., S.-C. Park, and J. Krug, 2009 Exploring the effect of sex on empirical fitness landscapes. The American Naturalist 174 Suppl 1: S15-30.

Denver, D. R., D. K. Howe, L. J. Wilhelm, C. A. Palmer, J. L. Anderson, K. C. Stein, P. C. Phillips, and S. Estes, 2010 Selective sweeps and parallel mutation in the adaptive recovery from deleterious mutation in Caenorhabditis elegans. Genome research 20: 1663-1671.

Denver, D. R., K. Morris, A. Kewalramani, K. E. Harris, A. Chow, S. Estes, M. Lynch, and W. K. Thomas, 2004a Abundance, distribution, and mutation rates of homopolymeric nucleotide runs in the genome of Caenorhabditis elegans. Journal of molecular evolution 58: 584-595.

Denver, D. R., K. Morris, M. Lynch, and W. K. Thomas, 2004b High mutation rate and predominance of insertions in the Caenorhabditis elegans nuclear genome. Nature 430: 679-682.

Diaz, S. A. and M. Viney, 2014 Genotypic-specific variance in Caenorhabditis elegans lifetime fecundity. Ecology and Evolution 4: 2058-2069.

Dohm, M. R., 2002 Repeatability Estimates Do Not Always Set an Upper Limit to Heritability. Functional Ecology 16: 273-280.

Dolgin, E. S., B. Charlesworth, S. E. Baird, and A. D. Cutter, 2007 Inbreeding and Outbreeding Depression in Caenorhabditis Nematodes. Evolution 61: 1339-1352.

Doroszuk, A., L. B. Snoek, E. Fradin, J. Riksen, and J. Kammenga, 2009 A genome-wide library of CB4856/N2 introgression lines of Caenorhabditis elegans. Nucleic acids research 37: e110-e110.

Duveau, F. and M.-A. Félix, 2012 Role of pleiotropy in the evolution of a cryptic developmental variation in Caenorhabditis elegans. PLoS biology 10: e1001230.

Estes, S., 2004 Mutation Accumulation in Populations of Varying Size: The Distribution of Mutational Effects for Fitness Correlates in Caenorhabditis elegans. Genetics 166: 1269-1279.

Estes, S., 2005 Spontaneous Mutational Correlations for Life-History, Morphological and Behavioral Characters in Caenorhabditis elegans. Genetics 170: 645-653.

Estes, S. and M. Lynch, 2003 Rapid Fitness Recovery in Muta- 
bioRxiv preprint doi: https://doi.org/10.1101/120865; this version posted March 27, 2017. The copyright holder for this preprint (which was not certified by peer review) is the author/funder, who has granted bioRxiv a license to display the preprint in perpetuity. It is made available under

tionally Degraded Lines of Caenorhabditis elegans. Evolution ${ }_{1541}$ 57: 1022-1030.

1542 Estes, S., P. C. Phillips, and D. R. Denver, 2011 FITNESS RECOV- 1543 ERY AND COMPENSATORY EVOLUTION IN NATURAL 1544 MUTANT LINES OF C. ELEGANS. Evolution 65: 2335-2344. 1545

Etienne, V., E. C. Andersen, J. M. Ponciano, D. Blanton, A. Ca- 1546 david, J. Joyner-Matos, C. Matsuba, B. Tabman, and C. F. Baer, 1547 2015 The red death meets the abdominal bristle: polygenic mu- 1548 tation for susceptibility to a bacterial pathogen in Caenorhabdi- 1549 tis elegans. Evolution 69: 508-519.

1550

Falconer, D. S., 1981 Introduction to quantitative genetics. Longman 1551 Publishing Group.

Farhadifar, R., J. M. Ponciano, E. C. Andersen, D. J. Needle- 1553 man, and C. F. Baer, 2016 Mutation Is a Sufficient and Robust ${ }_{1554}$ Predictor of Genetic Variation for Mitotic Spindle Traits in 1555 Caenorhabditis elegans. Genetics 203: 1859-1870.

Félix, M.-A. and M. Barkoulas, 2015 Pervasive robustness in ${ }^{1557}$ biological systems. Nature Reviews Genetics 16: 483-496. $\quad{ }_{1558}$

Fisher, R. A., 1930 The Genetical Theory of Natural Selection. A 1559 Complete Variorum Edition, Oxford University Press. 1560 Forsberg, S. K. G., J. S. Bloom, M. J. Sadhu, L. Kruglyak, and ${ }_{1561}$ Ö. Carlborg, 2017 Accounting for genetic interactions im- 1562 proves modeling of individual quantitative trait phenotypes 1563 in yeast. Nature Genetics 139: 1455.

Gaertner, B. E., M. D. Parmenter, M. V. Rockman, L. Kruglyak, 1565 and P. C. Phillips, 2012 More than the sum of its parts: a com- 1566 plex epistatic network underlies natural variation in thermal 1567 preference behavior in Caenorhabditis elegans. Genetics 192: 1568 1533-1542.

1569

Galton, F., 1886 Regression Towards Mediocrity in Hereditary 1570 Stature. The Journal of the Anthropological Institute of Great 1571 Britain and Ireland 15: 246.

1572

Gems, D. and D. L. Riddle, 2000 Defining wild-type life span in 1573 Caenorhabditis elegans. The journals of gerontology. Series A, 1574 Biological sciences and medical sciences 55: B215-9.

Ghosh, R., E. C. Andersen, J. A. Shapiro, J. P. Gerke, and 1576 L. Kruglyak, 2012 Natural variation in a chloride channel 1577 subunit confers avermectin resistance in C. elegans. Science 1578 (New York, N.Y.) 335: 574-578.

Gimond, C., R. Jovelin, S. Han, C. Ferrari, A. D. Cutter, and 1580 C. Braendle, 2013 Outbreeding depression with low genetic 1581 variation in selfing Caenorhabditis nematodes. Evolution 67: 1582 3087-3101.

Gloria-Soria, A. and R. B. R. Azevedo, 2008 npr-1 Regulates forag- 1584 ing and dispersal strategies in Caenorhabditis elegans. Current 1585 biology : CB 18: 1694-1699.

Goddard, M. E., K. E. Kemper, I. M. MacLeod, A. J. Chamberlain, 1587 and B. J. Hayes, 2016 Genetics of complex traits: prediction 1588 of phenotype, identification of causal polymorphisms and 1589 genetic architecture. Proc. R. Soc. B 283: 20160569.

1590

Gray, J. C. and A. D. Cutter, 2014 Mainstreaming Caenorhabdi- 1591 tis elegans in experimental evolution. Proceedings. Biological 1592 sciences 281: 20133055-20133055.

1593

Greene, J. S., M. Brown, M. Dobosiewicz, I. G. Ishida, E. Z. Ma- 1594 cosko, X. Zhang, R. A. Butcher, D. J. Cline, P. T. McGrath, 1595 and C. I. Bargmann, 2016 Balancing selection shapes density- 1596 dependent foraging behaviour. Nature 539: 254-258.

1597

Gruber, J. D., K. Vogel, G. Kalay, and P. J. Wittkopp, 2012 Con- 1598 trasting properties of gene-specific regulatory, coding, and 1599 copy number mutations in Saccharomyces cerevisiae: frequency, 1600 effects, and dominance. PLOS Genetics 8: e1002497.

1601 Gutteling, E. W., A. Doroszuk, J. A. G. Riksen, Z. Prokop, 1602
J. Reszka, and J. E. Kammenga, 2007 Environmental influence on the genetic correlations between life-history traits in Caenorhabditis elegans. Heredity 98: 206-213.

Halligan, D. L. and P. D. Keightley, 2009 Spontaneous Mutation Accumulation Studies in Evolutionary Genetics. Annual Review of Ecology, Evolution, and Systematics 40: 151-172.

Hansen, T. F., 2013 WHY EPISTASIS IS IMPORTANT FOR SELECTION AND ADAPTATION 67: 3501-3511.

Hayes, J. P. and S. H. Jenkins, 1997 Individual Variation in Mammals. Journal of Mammalogy 78: 274-293.

Hemani, G., S. Knott, and C. Haley, 2013 An Evolutionary Perspective on Epistasis and the Missing Heritability. PLOS Genetics 9: e1003295.

Henderson, C. R., 1975 Best Linear Unbiased Estimation and Prediction under a Selection Model 31: 423.

Henderson, C. R., 1985 Best Linear Unbiased Prediction of Nonadditive Genetic Merits in Noninbred Populations. Journal of animal science 60: 111-117.

Hill, W. G., 1982 Rates of change in quantitative traits from fixation of new mutations. Proceedings of the National Academy of Sciences 79: 142-145.

Hill, W. G., M. E. Goddard, and P. M. Visscher, 2008 Data and Theory Point to Mainly Additive Genetic Variance for Complex Traits 4: e1000008.

Hirsh, D., D. Oppenheim, and M. Klass, 1976 Development of the reproductive system of Caenorhabditis elegans. Developmental biology 49: 200-219.

Huang, A., S. Xu, and X. Cai, 2014 Whole-genome quantitative trait locus mapping reveals major role of epistasis on yield of rice. PloS one 9: e87330.

Huang, B. E., A. W. George, K. L. Forrest, A. Kilian, M. J. Hayden, M. K. Morell, and C. R. Cavanagh, 2012 A multiparent advanced generation inter-cross population for genetic analysis in wheat. Plant biotechnology journal 10: 826-839.

Jiang, Y. and J. C. Reif, 2015 Modeling Epistasis in Genomic Selection. Genetics 201: 759-768.

Jombart, T., 2008 adegenet: a R package for the multivariate analysis of genetic markers. Bioinformatics (Oxford, England) 24: 1403-1405.

Jombart, T., S. Devillard, and F. Balloux, 2010 Discriminant analysis of principal components: a new method for the analysis of genetically structured populations. BMC genetics 11: 94 .

Joyner-Matos, J., A. Upadhyay, M. P. Salomon, V. Grigaltchik, and C. F. Baer, 2009 Genetic (Co)variation for life span in rhabditid nematodes: role of mutation, selection, and history. The journals of gerontology. Series A, Biological sciences and medical sciences 64: 1134-1145.

Kamran-Disfani, A. and A. F. Agrawal, 2014 Selfing, adaptation and background selection in finite populations. Journal of evolutionary biology 27: 1360-1371.

Kaur, T. and M. V. Rockman, 2014 Crossover heterogeneity in the absence of hotspots in Caenorhabditis elegans. Genetics 196: 137-148.

King, E. G., S. J. Macdonald, and A. D. Long, 2012 Properties and power of the Drosophila Synthetic Population Resource for the routine dissection of complex traits. Genetics 191: 935-949.

King, E. G., B. J. Sanderson, C. L. McNeil, A. D. Long, and S. J. Macdonald, 2014 Genetic dissection of the Drosophila melanogaster female head transcriptome reveals widespread allelic heterogeneity. PLOS Genetics 10: e1004322.

Knight, C. G., R. B. Azevedo, and A. M. Leroi, 2001 Testing life-history pleiotropy in Caenorhabditis elegans. 55: 1795-1804. 
bioRxiv preprint doi: https://doi.org/10.1101/120865; this version posted March 27, 2017. The copyright holder for this preprint (which was not certified by peer review) is the author/funder, who has granted bioRxiv a license to display the preprint in perpetuity. It is made available under

Kover, P. X., W. Valdar, J. Trakalo, N. Scarcelli, I. M. Ehrenreich, 1665 M. D. Purugganan, C. Durrant, and R. Mott, 2009 A Multipar- 1666 ent Advanced Generation Inter-Cross to Fine-Map Quantita- 1667 tive Traits in Arabidopsis thaliana. PLOS Genetics 5: e1000551. 1668 Lessells, C. M. and P. T. Boag, 1987 Unrepeatable Repeatabilities: 1669 A Common Mistake. The Auk 104: 116-121.

1670

Li, H. and R. Durbin, 2010 Fast and accurate long-read align- 1671 ment with Burrows-Wheeler transform. Bioinformatics (Ox- 1672 ford, England) 26: 589-595.

1673

Lipinski, K. J., J. C. Farslow, K. A. Fitzpatrick, M. Lynch, V. Katju, 1674 and U. Bergthorsson, 2011 High Spontaneous Rate of Gene 1675 Duplication in Caenorhabditis elegans. Current Biology 21: 306- 1676 310.

1677

Long, A. D., S. J. Macdonald, and E. G. King, 2014 Dissecting 1678 complex traits using the Drosophila Synthetic Population Re- 1679 source. Trends in genetics : TIG 30: 488-495.

Lynch, M. and B. Walsh, 1998 Genetics and Analysis of Quantitative 1681 Traits. Sinauer Associates Incorporated.

1682

Macdonald, S. J. and A. D. Long, 2007 Joint estimates of quanti- 1683 tative trait locus effect and frequency using synthetic recom- 1684 binant populations of Drosophila melanogaster. Genetics 176: 1685 1261-1281.

Mackay, I. J., P. Bansept-Basler, T. Barber, A. R. Bentley, J. Cock- 1687 ram, N. Gosman, A. J. Greenland, R. Horsnell, R. Howells, 1688 D. M. O'Sullivan, G. A. Rose, and P. J. Howell, 2014 An eight- 1689 parent multiparent advanced generation inter-cross popula- 1690 tion for winter-sown wheat: creation, properties, and valida- 1691 tion. G3 (Bethesda, Md.) 4: 1603-1610.

1692

Malmberg, R. L., S. Held, A. Waits, and R. Mauricio, 2005 Epista- 1693 sis for fitness-related quantitative traits in Arabidopsis thaliana 1694 grown in the field and in the greenhouse. Genetics 171: 2013- 1695 2027.

Manolio, T. A., F. S. Collins, N. J. Cox, D. B. Goldstein, L. A. ${ }_{1697}$ Hindorff, D. J. Hunter, M. I. McCarthy, E. M. Ramos, L. R. 1698 Cardon, A. Chakravarti, J. H. Cho, A. E. Guttmacher, A. Kong, 1699 L. Kruglyak, E. Mardis, C. N. Rotimi, M. Slatkin, D. Valle, A. S. 1700 Whittemore, M. Boehnke, A. G. Clark, E. E. Eichler, G. Gibson, 1701 J. L. Haines, T. F. C. Mackay, S. A. McCarroll, and P. M. Viss- 1702 cher, 2009 Finding the missing heritability of complex diseases. 1703 461: 747-753.

1704

Marouli, E., M. Graff, C. Medina-Gomez, K. S. Lo, A. R. Wood, 1705 T. R. Kjaer, R. S. Fine, Y. Lu, C. Schurmann, H. M. Highland, 1706 S. Rüeger, G. Thorleifsson, A. E. Justice, D. Lamparter, K. E. 1707 Stirrups, V. Turcot, K. L. Young, T. W. Winkler, T. Esko, T. Ka- 1708 raderi, A. E. Locke, N. G. D. Masca, M. C. Y. Ng, P. Mudgal, 1709 M. A. Rivas, S. Vedantam, A. Mahajan, X. Guo, G. Abeca- 1710 sis, K. K. Aben, L. S. Adair, D. S. Alam, E. Albrecht, K. H. 1711 Allin, M. Allison, P. Amouyel, E. V. Appel, D. Arveiler, 1712 F. W. Asselbergs, P. L. Auer, B. Balkau, B. Banas, L. E. Bang, 1713 M. Benn, S. Bergmann, L. F. Bielak, M. Blüher, H. Boeing, 1714 E. Boerwinkle, C. A. Böger, L. L. Bonnycastle, J. Bork-Jensen, 1715 M. L. Bots, E. P. Bottinger, D. W. Bowden, I. Brandslund, 1716 G. Breen, M. H. Brilliant, L. Broer, A. A. Burt, A. S. Butter- 1717 worth, D. J. Carey, M. J. Caulfield, J. C. Chambers, D. I. Chas- 1718 man, Y.-D. I. Chen, R. Chowdhury, C. Christensen, A. Y. Chu, 1719 M. Cocca, F. S. Collins, J. P. Cook, J. Corley, J. C. Galbany, 1720 A. J. Cox, G. Cuellar-Partida, J. Danesh, G. Davies, P. I. W. 1721 de Bakker, G. J. de Borst, S. de Denus, M. C. H. de Groot, 1722 R. de Mutsert, I. J. Deary, G. Dedoussis, E. W. Demerath, A. I. 1723 den Hollander, J. G. Dennis, E. Di Angelantonio, F. Drenos, 1724 M. Du, A. M. Dunning, D. F. Easton, T. Ebeling, T. L. Ed- 1725 wards, P. T. Ellinor, P. Elliott, E. Evangelou, A.-E. Farmaki, 1726
J. D. Faul, M. F. Feitosa, S. Feng, E. Ferrannini, M. M. Ferrario, J. Ferrieres, J. C. Florez, I. Ford, M. Fornage, P. W. Franks, R. Frikke-Schmidt, T. E. Galesloot, W. Gan, I. Gandin, P. Gasparini, V. Giedraitis, A. Giri, G. Girotto, S. D. Gordon, P. Gordon-Larsen, M. Gorski, N. Grarup, M. L. Grove, V. Gudnason, S. Gustafsson, T. Hansen, K. M. Harris, T. B. Harris, A. T. Hattersley, C. Hayward, L. He, I. M. Heid, K. Heikkilä, Ø. Helgeland, J. Hernesniemi, A. W. Hewitt, L. J. Hocking, M. Hollensted, O. L. Holmen, G. K. Hovingh, J. M. M. Howson, C. B. Hoyng, P. L. Huang, K. Hveem, M. A. Ikram, E. Ingelsson, A. U. Jackson, J.-H. Jansson, G. P. Jarvik, G. B. Jensen, M. A. Jhun, Y. Jia, X. Jiang, S. Johansson, M. E. Jørgensen, T. Jørgensen, P. Jousilahti, J. W. Jukema, B. Kahali, R. S. Kahn, M. Kähönen, P. R. Kamstrup, S. Kanoni, J. Kaprio, M. Karaleftheri, S. L. R. Kardia, F. Karpe, F. Kee, R. Keeman, L. A. Kiemeney, H. Kitajima, K. B. Kluivers, T. Kocher, P. Komulainen, J. Kontto, J. S. Kooner, C. Kooperberg, P. Kovacs, J. Kriebel, H. Kuivaniemi, S. Küry, J. Kuusisto, M. La Bianca, M. Laakso, T. A. Lakka, E. M. Lange, L. A. Lange, C. D. Langefeld, C. Langenberg, E. B. Larson, I.-T. Lee, T. Lehtimäki, C. E. Lewis, H. Li, J. Li, R. Li-Gao, H. Lin, L.-A. Lin, X. Lin, L. Lind, J. Lindström, A. Linneberg, Y. Liu, Y. Liu, A. Lophatananon, J. Luan, S. A. Lubitz, L.-P. Lyytikäinen, D. A. Mackey, P. A. F. Madden, A. K. Manning, S. Männistö, G. Marenne, J. Marten, N. G. Martin, A. L. Mazul, K. Meidtner, A. Metspalu, P. Mitchell, K. L. Mohlke, D. O. Mook-Kanamori, A. Morgan, A. D. Morris, A. P. Morris, M. Müller-Nurasyid, P. B. Munroe, M. A. Nalls, and M. Nauck, 2017 Rare and lowfrequency coding variants alter human adult height. Nature 542: 186-190.

Masri, L., R. D. Schulte, N. Timmermeyer, S. Thanisch, L. L. Crummenerl, G. Jansen, N. K. Michiels, and H. Schulenburg, 2013 Sex differences in host defence interfere with parasitemediated selection for outcrossing during host-parasite coevolution. Ecology letters 16: 461-468.

Matuszewski, S., J. Hermisson, and M. Kopp, 2015 Catch Me if You Can: Adaptation from Standing Genetic Variation to a Moving Phenotypic Optimum. Genetics 200: 1255-1274.

Maupas, E., 1900 Modes et formes de reproduction des nematodes. Archives de zoologie expérimentale et générale pp. 463-624.

McGrath, P. T., M. V. Rockman, M. Zimmer, H. Jang, E. Z. Macosko, L. Kruglyak, and C. I. Bargmann, 2009 Quantitative mapping of a digenic behavioral trait implicates globin variation in C. elegans sensory behaviors. Neuron 61: 692-699.

McKenna, A., M. Hanna, E. Banks, A. Sivachenko, K. Cibulskis, A. Kernytsky, K. Garimella, D. Altshuler, S. Gabriel, M. Daly, and M. A. DePristo, 2010 The Genome Analysis Toolkit: a MapReduce framework for analyzing next-generation DNA sequencing data. Genome research 20: 1297-1303.

McMullen, M. D., S. Kresovich, H. S. Villeda, P. Bradbury, H. Li, Q. Sun, S. Flint-Garcia, J. Thornsberry, C. Acharya, C. Bottoms, P. Brown, C. Browne, M. Eller, K. Guill, C. Harjes, D. Kroon, N. Lepak, S. E. Mitchell, B. Peterson, G. Pressoir, S. Romero, M. O. Rosas, S. Salvo, H. Yates, M. Hanson, E. Jones, S. Smith, J. C. Glaubitz, p. M. p. Goodman, D. Ware, J. B. Holland, and E. S. Buckler, 2009 Genetic Properties of the Maize Nested Association Mapping Population. Science (New York, N.Y.) 325: 737-740.

Meier, B., S. L. Cooke, J. Weiss, A. P. Bailly, L. B. Alexandrov, J. Marshall, K. Raine, M. Maddison, E. Anderson, M. R. Stratton, A. Gartner, and P. J. Campbell, 2014 C. elegans whole- 
bioRxiv preprint doi: https://doi.org/10.1101/120865; this version posted March 27, 2017. The copyright holder for this preprint (which was not certified by peer review) is the author/funder, who has granted bioRxiv a license to display the preprint in perpetuity. It is made available under

genome sequencing reveals mutational signatures related to 1789 carcinogens and DNA repair deficiency. Genome research 24: 1790 1624-1636.

Meuwissen, T. and M. Goddard, 2010 Accurate prediction of 1792 genetic values for complex traits by whole-genome resequenc- 1793 ing. Genetics 185: 623-631.

1794

Meuwissen, T. H., B. J. Hayes, and M. E. Goddard, 2001 Predic- 1795 tion of total genetic value using genome-wide dense marker 1796 maps. Genetics 157: 1819-1829.

Monnahan, P. J. and J. K. Kelly, 2015a Epistasis Is a Major Deter- 1798 minant of the Additive Genetic Variance in Mimulus guttatus. 1799 PLOS Genetics 11: e1005201.

Monnahan, P. J. and J. K. Kelly, 2015b Naturally segregating loci 1801 exhibit epistasis for fitness. Biology Letters 11: $20150498 . \quad 1802$

Morran, L. T., M. D. Parmenter, and P. C. Phillips, 2009 Muta- 1803 tion load and rapid adaptation favour outcrossing over self- 1804 fertilization. Nature 462: 350-352.

MUKAI, T., 1967 Synergistic interaction of spontaneous mutant poly- 1806 genes controlling viability in Drosophila melanogaster. Genetics. 1807

Murray, R. L., J. L. Kozlowska, and A. D. Cutter, 2011 Heritable 1808 determinants of male fertilization success in the nematode 1809 Caenorhabditis elegans. BMC evolutionary biology 11: $99 . \quad 1810$

Neher, R. A. and B. I. Shraiman, 2009 Competition between 1811 recombination and epistasis can cause a transition from allele 1812 to genotype selection. Proceedings of the National Academy 1813 of Sciences of the United States of America 106: 6866-6871. $\quad 1814$

Nigon, V., 1949 Les modalites de la reproduction et le deter- 1815 minisme du sexe chez quelques nematodes libres. Annales de 1816 Sciences Naturelles - Zool. Biol. Anim. 11: 1-132.

Noble, L. M., A. S. Chang, D. McNelis, M. Kramer, M. Yen, 1818 J. P. Nicodemus, D. D. Riccardi, P. Ammerman, M. Phillips, 1819 T. Islam, and M. V. Rockman, 2015 Natural Variation in plep-1 ${ }_{1820}$ Causes Male-Male Copulatory Behavior in C. elegans. Current ${ }_{1821}$ biology : CB 25: 2730-2737.

Paaby, A. B., A. G. White, D. D. Riccardi, K. C. Gunsalus, F. Piano, 1823 and M. V. Rockman, 2015 Wild worm embryogenesis harbors 1824 ubiquitous polygenic modifier variation. eLife 4: 1061.

Pascual, L., N. Desplat, B. E. Huang, A. Desgroux, L. Bruguier, 1826 J.-P. Bouchet, Q. H. Le, B. Chauchard, P. Verschave, and 1827 M. Causse, 2015 Potential of a tomato MAGIC population 1828 to decipher the genetic control of quantitative traits and detect ${ }_{1829}$ causal variants in the resequencing era. Plant biotechnology 1830 journal 13: 565-577.

1831

Philip, V. M., G. Sokoloff, C. L. Ackert-Bicknell, M. Striz, 1832 L. Branstetter, M. A. Beckmann, J. S. Spence, B. L. Jackson, 1833 L. D. Galloway, P. Barker, A. M. Wymore, P. R. Hunsicker, 1834 D. C. Durtschi, G. S. Shaw, S. Shinpock, K. F. Manly, D. R. ${ }_{1835}$ Miller, K. D. Donohue, C. T. Culiat, G. A. Churchill, W. R. Lar- 1836 iviere, A. A. Palmer, B. F. O'Hara, B. H. Voy, and E. J. Chesler, ${ }_{1837}$ 2011 Genetic analysis in the Collaborative Cross breeding 1838 population. Genome research 21: 1223-1238.

1839

Phillips, N., M. Salomon, A. Custer, D. Ostrow, and C. F. 1840 Baer, 2009 Spontaneous mutational and standing genetic 1841 (co)variation at dinucleotide microsatellites in Caenorhabditis 1842 briggsae and Caenorhabditis elegans. Molecular biology and 1843 evolution 26: 659-669.

Phillips, P. C., 2008 Epistasis - the essential role of gene interac- 1845 tions in the structure and evolution of genetic systems. Nature 1846 Reviews Genetics 9: 855-867.

Phillips, P. C., S. P. Otto, and M. C. Whitlock, 2000 Beyond the 1848 average. In Epistasis and the Evolutionary Process, edited by J. B. 1849 Wolf, E. D. Brodie, and M. J. Wade, Oxford University Press. ${ }_{1850}$
Poelwijk, F. J., S. Tănase-Nicola, D. J. Kiviet, and S. J. Tans, 2011 Reciprocal sign epistasis is a necessary condition for multipeaked fitness landscapes. Journal of theoretical biology 272: 141-144.

Poullet, N., A. Vielle, C. Gimond, S. Carvalho, H. Teotónio, and C. Braendle, 2016 Complex heterochrony underlies the evolution of Caenorhabditis elegans hermaphrodite sex allocation 70: 2357-2369.

Pritchard, J. K., 2002 The allelic architecture of human disease genes: common disease-common variant... or not? Human molecular genetics 11: 2417-2423.

Reddy, K. C., E. C. Andersen, L. Kruglyak, and D. H. Kim, 2009 A polymorphism in $n p r-1$ is a behavioral determinant of pathogen susceptibility in C. elegans. Science (New York, N.Y.) 323: 382-384.

Robinson, G. K., 1991 That BLUP is a good thing: the estimation of random effects. Statistical science .

Rockman, M. V. and L. Kruglyak, 2008 Breeding designs for recombinant inbred advanced intercross lines. Genetics 179: 1069-1078.

Rockman, M. V. and L. Kruglyak, 2009 Recombinational landscape and population genomics of Caenorhabditis elegans. PLOS Genetics 5: e1000419.

Rockman, M. V., S. S. Skrovanek, and L. Kruglyak, 2010 Selection at linked sites shapes heritable phenotypic variation in $C$. elegans. Science (New York, N.Y.) 330: 372-376.

Ruby, J. G., C. Jan, C. Player, M. J. Axtell, W. Lee, C. Nusbaum, H. Ge, and D. P. Bartel, 2006 Large-scale sequencing reveals 21U-RNAs and additional microRNAs and endogenous siRNAs in C. elegans. Cell 127: 1193-1207.

Schoustra, S., S. Hwang, J. Krug, and J. A. G. M. de Visser, 2016 Diminishing-returns epistasis among random beneficial mutations in a multicellular fungus. Proc. R. Soc. B 283: 20161376.

Seidel, H. S., M. Ailion, J. Li, A. van Oudenaarden, M. V. Rockman, and L. Kruglyak, 2011 A novel sperm-delivered toxin causes late-stage embryo lethality and transmission ratio distortion in C. elegans. PLoS biology 9: e1001115.

Seidel, H. S., M. V. Rockman, and L. Kruglyak, 2008 Widespread genetic incompatibility in C. elegans maintained by balancing selection. Science (New York, N.Y.) 319: 589-594.

Seyfert, A. L., M. E. A. Cristescu, L. Frisse, S. Schaack, W. K. Thomas, and M. Lynch, 2008 The Rate and Spectrum of Microsatellite Mutation in Caenorhabditis elegans and Daphnia pulex. Genetics 178: 2113-2121.

Shao, H., L. C. Burrage, D. S. Sinasac, A. E. Hill, S. R. Ernest, W. O’Brien, H.-W. Courtland, K. J. Jepsen, A. Kirby, E. J. Kulbokas, M. J. Daly, K. W. Broman, E. S. Lander, and J. H. Nadeau, 2008 Genetic architecture of complex traits: large phenotypic effects and pervasive epistasis. 105: 19910-19914. Shen, X., M. Alam, L. Ronnegard, and M. X. Shen, 2014 Package 'hglm'.

Simon, M., O. Loudet, S. Durand, A. Bérard, D. Brunel, F.-X. Sennesal, M. Durand-Tardif, G. Pelletier, and C. Camilleri, 2008 Quantitative trait loci mapping in five new large recombinant inbred line populations of Arabidopsis thaliana genotyped with consensus single-nucleotide polymorphism markers. Genetics 178: 2253-2264.

Sokal, R. R. and F. J. Rohlf, 1995 Biometry: the principles and practice of statistics in biological sciences. WH Free Company.

Speed, D. and D. J. Balding, 2015 Relatedness in the postgenomic era: is it still useful? Nature Reviews Genetics 16: 33-44. 
bioRxiv preprint doi: https://doi.org/10.1101/120865; this version posted March 27, 2017. The copyright holder for this preprint (which was not certified by peer review) is the author/funder, who has granted bioRxiv a license to display the preprint in perpetuity. It is made available under

Speed, D., N. Cai, T. U. Consortium, M. Johnson, S. Nejentsev, 1913 and D. Balding, 2016 Re-evaluation of SNP heritability in 1914 complex human traits. bioRxiv p. 074310.

Speed, D., G. Hemani, M. R. Johnson, and D. J. Balding, 20121916 Improved heritability estimation from genome-wide SNPs. 1917 American journal of human genetics 91: 1011-1021. 1918

Sterken, M. G., L. B. Snoek, J. E. Kammenga, and E. C. Andersen, 1919 2015 The laboratory domestication of Caenorhabditis elegans. 1920 Trends in genetics : TIG 31: 224-231.

1921

Stewart, A. D. and P. C. Phillips, 2002 Selection and maintenance 1922 of androdioecy in Caenorhabditis elegans. Genetics 160: 975- 1923 982.

Stiernagle, T., 2006 Maintenance of C. elegans. WormBook : the 1925 online review of C. elegans biology pp. 1-11.

1926

Swierczek, N. A., A. C. Giles, C. H. Rankin, and R. A. Kerr, 20111927 High-throughput behavioral analysis in C. elegans. Nature 1928 methods 8: 592-598.

1929

Teotónio, H., S. Carvalho, D. Manoel, M. Roque, and I. M. Chelo, 1930 2012 Evolution of outcrossing in experimental populations of 1931 Caenorhabditis elegans. PloS one 7: e35811.

1932

Teotónio, H., S. Estes, P. C. Phillips, and C. F. Baer, 2017 Evolu- 1933 tion experiments with Caenorhabditis nematodes. Genetics 1934

1935

Teotónio, H., D. Manoel, and P. C. Phillips, 2006 Genetic Varia- 1936 tion for Outcrossing among Caenorhabditis elegans Isolates 60: 1937 1300-1305.

Theologidis, I., I. M. Chelo, C. Goy, and H. Teotónio, 2014 Re- 1939 productive assurance drives transitions to self-fertilization in 1940 experimental Caenorhabditis elegans. BMC biology 12: 93.

Thepot, S., G. Restoux, I. Goldringer, F. Hospital, D. Gouache, 1942 I. Mackay, and J. Enjalbert, 2015 Efficiently Tracking Selection 1943 in a Multiparental Population: The Case of Earliness in Wheat. 1944 Genetics 199: 609-623.

Thompson, O. A., L. B. Snoek, H. Nijveen, M. G. Sterken, 1946 R. J. M. Volkers, R. Brenchley, A. Van't Hof, R. P. J. Bevers, 1947 A. R. Cossins, I. Yanai, A. Hajnal, T. Schmid, J. D. Perkins, 1948 D. Spencer, L. Kruglyak, E. C. Andersen, D. G. Moerman, L. W. 1949 Hillier, J. E. Kammenga, and R. H. Waterston, 2015 Remark- 1950 ably Divergent Regions Punctuate the Genome Assembly of 1951 the Caenorhabditis elegans Hawaiian Strain CB4856. Genetics 1952 200: 975-989.

Tyler, A. L., L. R. Donahue, G. A. Churchill, and G. W. Carter, 1954 2016 Weak Epistasis Generally Stabilizes Phenotypes in a 1955 Mouse Intercross. PLOS Genetics 12: e1005805.

1956

Valdar, W., L. C. Solberg, D. Gauguier, S. Burnett, P. Klenerman, 1957 W. O. Cookson, M. S. Taylor, J. N. P. Rawlins, R. Mott, and 1958 J. Flint, 2006 Genome-wide genetic association of complex 1959 traits in heterogeneous stock mice. Nature Genetics 38: 879- 1960 887.

1961

Vanhaeren, H., N. Gonzalez, F. Coppens, L. De Milde, 1962 T. Van Daele, M. Vermeersch, N. B. Eloy, V. Storme, and D. Inzé, 1963 2014 Combining growth-promoting genes leads to positive 1964 epistasis in Arabidopsis thaliana. eLife 3: e02252.

1965

VanRaden, P. M., 2008 Efficient methods to compute genomic 1966 predictions. Journal of dairy science 91: 4414-4423.

Visscher, P. M., G. Hemani, A. A. E. Vinkhuyzen, G.-B. Chen, S. H. 1968 Lee, N. R. Wray, M. E. Goddard, and J. Yang, 2014 Statistical 1969 power to detect genetic (co)variance of complex traits using 1970 SNP data in unrelated samples. PLOS Genetics 10: e1004269. 1971 Visscher, P. M., B. McEVOY, and J. Yang, 2010 From Galton 1972 to GWAS: quantitative genetics of human height. Genetics 1973 Research 92: 371-379.
Weigel, D., 2012 Natural variation in Arabidopsis: from molecular genetics to ecological genomics. Plant physiology 158: 2-22.

Weinreich, D. M., Y. Lan, C. S. Wylie, and R. B. Heckendorn, 2013 Should evolutionary geneticists worry about higher-order epistasis? Current opinion in genetics \& development 23: 700-707.

Weinreich, D. M., R. A. Watson, and L. Chao, 2005 Perspective: Sign epistasis and genetic constraint on evolutionary trajectories. Evolution 59: 1165-1174.

Whitlock, M. C. and D. Bourguet, 2000 FACTORS AFFECTING THE GENETIC LOAD IN DROSOPHILA: SYNERGISTIC EPISTASIS AND CORRELATIONS AMONG FITNESS COMPONENTS. Evolution 54: 1654.

Whitlock, M. C., P. C. Phillips, F. B. G. Moore, and S. J. Tonsor, 1995 Multiple Fitness Peaks and Epistasis. Annual Review of Ecology and Systematics 26: 601-629.

Wolf, J. B., E. D. Brodie, and M. J. Wade, editors, 2000 Epistasis and the Evolutionary Process. Oxford University Press.

Wood, A. R., T. Esko, J. Yang, S. Vedantam, T. H. Pers, S. Gustafsson, A. Y. Chu, K. Estrada, J. Luan, Z. Kutalik, N. Amin, M. L. Buchkovich, D. C. Croteau-Chonka, F. R. Day, Y. Duan, T. Fall, R. Fehrmann, T. Ferreira, A. U. Jackson, J. Karjalainen, K. S. Lo, A. E. Locke, R. Mägi, E. Mihailov, E. Porcu, J. C. Randall, A. Scherag, A. A. E. Vinkhuyzen, H.-J. Westra, T. W. Winkler, T. Workalemahu, J. H. Zhao, D. Absher, E. Albrecht, D. Anderson, J. Baron, M. Beekman, A. Demirkan, G. B. Ehret, B. Feenstra, M. F. Feitosa, K. Fischer, R. M. Fraser, A. Goel, J. Gong, A. E. Justice, S. Kanoni, M. E. Kleber, K. Kristiansson, U. Lim, V. Lotay, J. C. Lui, M. Mangino, I. Mateo Leach, C. Medina-Gomez, M. A. Nalls, D. R. Nyholt, C. D. Palmer, D. Pasko, S. Pechlivanis, I. Prokopenko, J. S. Ried, S. Ripke, D. Shungin, A. Stancáková, R. J. Strawbridge, Y. J. Sung, T. Tanaka, A. Teumer, S. Trompet, S. W. van der Laan, J. van Setten, J. V. van Vliet-Ostaptchouk, Z. Wang, L. Yengo, W. Zhang, U. Afzal, J. Arnlöv, G. M. Arscott, S. Bandinelli, A. Barrett, C. Bellis, A. J. Bennett, C. Berne, M. Blüher, J. L. Bolton, Y. Böttcher, H. A. Boyd, M. Bruinenberg, B. M. Buckley, S. Buyske, I. H. Caspersen, P. S. Chines, R. Clarke, S. ClaudiBoehm, M. Cooper, E. W. Daw, P. A. De Jong, J. Deelen, G. Delgado, J. C. Denny, R. Dhonukshe-Rutten, M. Dimitriou, A. S. F. Doney, M. Dörr, N. Eklund, E. Eury, L. Folkersen, M. E. Garcia, F. Geller, V. Giedraitis, A. S. Go, H. Grallert, T. B. Grammer, J. Gräßler, H. Grönberg, L. C. P. G. M. de Groot, C. J. Groves, J. Haessler, P. Hall, T. Haller, G. Hallmans, A. Hannemann, C. A. Hartman, M. Hassinen, C. Hayward, N. L. Heard-Costa, Q. Helmer, G. Hemani, A. K. Henders, H. L. Hillege, M. A. Hlatky, W. Hoffmann, P. Hoffmann, O. Holmen, J. J. HouwingDuistermaat, T. Illig, A. Isaacs, A. L. James, J. Jeff, B. Johansen, Å. Johansson, J. Jolley, T. Juliusdottir, J. Junttila, A. N. Kho, L. Kinnunen, N. Klopp, T. Kocher, W. Kratzer, P. Lichtner, L. Lind, J. Lindström, S. Lobbens, M. Lorentzon, Y. Lu, V. Lyssenko, P. K. E. Magnusson, A. Mahajan, M. Maillard, W. L. McArdle, C. A. McKenzie, S. McLachlan, P. J. McLaren, C. Menni, S. Merger, L. Milani, A. Moayyeri, K. L. Monda, M. A. Morken, G. Müller, M. Müller-Nurasyid, A. W. Musk, N. Narisu, M. Nauck, I. M. Nolte, M. M. Nöthen, L. Oozageer, S. Pilz, N. W. Rayner, F. Renström, N. R. Robertson, L. M. Rose, R. Roussel, S. Sanna, H. Scharnagl, S. Scholtens, F. R. Schumacher, H. Schunkert, R. A. Scott, J. Sehmi, T. Seufferlein, J. Shi, K. Silventoinen, J. H. Smit, A. V. Smith, J. Smolonska, A. V. Stanton, K. Stirrups, D. J. Stott, H. M. Stringham, J. Sund- 
bioRxiv preprint doi: https://doi org/10.1101/120865; this version posted March 27, 2017. The copyright holder for this preprint (which was not certified by peer review) is the author/funder, who has granted bioRxiv a license to display the preprint in perpetuity. It is made available under

ström, M. A. Swertz, A.-C. Syvänen, B. O. Tayo, G. Thorleifsson, J. P. Tyrer, S. van Dijk, N. M. van Schoor, N. van der Velde, D. van Heemst, F. V. A. van Oort, S. H. Vermeulen, N. Verweij, J. M. Vonk, L. L. Waite, M. Waldenberger, R. Wennauer, L. R. Wilkens, C. Willenborg, T. Wilsgaard, M. K. Wojczynski, A. Wong, A. F. Wright, and Q. Zhang, 2014 Defining the role of common variation in the genomic and biological architecture of adult human height. Nature Genetics 46: 1173-1186.

Wray, G. A., 2007 The evolutionary significance of cis-regulatory mutations. Nature Reviews Genetics 8: 206-216.

Wright, S., 1932 The roles of mutation, inbreeding, crossbreeding, and selection in evolution.

Yang, J., B. Benyamin, B. P. McEvoy, S. Gordon, A. K. Henders, D. R. Nyholt, P. A. Madden, A. C. Heath, N. G. Martin, G. W. Montgomery, M. E. Goddard, and P. M. Visscher, 2010 Common SNPs explain a large proportion of the heritability for human height. Nature Genetics 42: 565-569.

Yang, J., T. A. Manolio, L. R. Pasquale, E. Boerwinkle, N. Caporaso, J. M. Cunningham, M. de Andrade, B. Feenstra, E. Feingold, M. G. Hayes, W. G. Hill, M. T. Landi, A. Alonso, G. Lettre, P. Lin, H. Ling, W. Lowe, R. A. Mathias, M. Melbye, E. Pugh, M. C. Cornelis, B. S. Weir, M. E. Goddard, and P. M. Visscher, 2011 Genome partitioning of genetic variation for complex traits using common SNPs. Nature Genetics 43: 519-525.

Zheng, C., M. P. Boer, and F. A. van Eeuwijk, 2015 Reconstruction of Genome Ancestry Blocks in Multiparental Populations. Genetics 200: 1073-1087.

Zheng, C., M. P Boer, and F. A. van Eeuwijk, 2014 A general modeling framework for genome ancestral origins in multiparental populations. Genetics 198: 87-101.

Zwarts, L., M. M. Magwire, M. A. Carbone, M. Versteven, L. Herteleer, R. R. H. Anholt, P. Callaerts, and T. F. C. Mackay, 2011 Complex genetic architecture of Drosophila aggressive behavior. Proceedings of the National Academy of Sciences of the United States of America 108: 17070-17075. 